\title{
1 Dendritic domain-specific sampling of long-range axons shapes \\ 2 feedforward and feedback connectivity of L5 neurons
}

4 Alessandro R. Galloni ${ }^{1,2}$, Zhiwen $\mathrm{Ye}^{1}$ and Ede Rancz ${ }^{1 *}$

$5{ }^{1}$ The Francis Crick Institute, London, United Kingdom; ${ }^{2}$ University College London, United Kingdom.

$6 \quad{ }^{*}$ For correspondence: ede.rancz@crick.ac.uk

7

8

\section{Abstract}

Feedforward and feedback pathways interact in specific dendritic domains to enable cognitive functions such as predictive processing and learning. Based on axonal projections, hierarchically lower areas are thought to form synapses primarily on dendrites in middle cortical layers, while higher-order areas are posited to target dendrites in layer 1 and in deep layers. However, the extent to which functional synapses form in regions of axo-dendritic overlap has not been extensively studied. Here, we use viral tracing in the secondary visual cortex of mice to map brain-wide inputs to thick-tufted layer 5 pyramidal neurons. Furthermore, we provide a comprehensive map of input locations through subcellular optogenetic circuit mapping. We show that input pathways target distinct dendritic domains with far greater specificity than appears from their axonal branching, often deviating substantially from the canonical patterns. Common assumptions regarding the dendrite-level interaction of feedforward and feedback inputs may thus need revisiting. 


\section{Introduction}

22 One of the key organizing principles of connectivity within the neocortex is thought to be hierarchy

23 between cortical areas. This notion was originally proposed by Hubel \& Wiesel (Hubel \& Wiesel 1962) to account for the increasing receptive field complexity in areas progressively further from the retina. In purely feedforward (FF) networks, such as artificial neural networks used successfully in computer vision (LeCun et al 2015), hierarchy is generally defined by synaptic distance from the sensory periphery. However, in highly recurrent networks like the cortex, it is not possible to apply this definition consistently beyond the initial levels. Instead, the laminar patterns of axonal projections are often used to deduce relative levels of hierarchy. For example, the projection from primary to secondary visual cortex, which is classically defined as $\mathrm{FF}$, is characterised by dense axon terminations in middle cortical layers (particularly L4). Meanwhile, the projection from secondary to primary visual cortex, used as the basis for defining feedback (FB), primarily targets L1 and to a lesser extent deeper layers (Rockland \& Pandya 1979). This pattern of FF and FB projections also appears in many other brain regions, and has been used as a proxy to describe the hierarchical relationships between a large number of areas across the brain (D'Souza et al 2016, D'Souza et al 2020, Felleman \& Van Essen 1991, Harris et al 2019, Wang et al 2020b, Zeng 2018).

Projections, however, do not guarantee functional connections. The link between the two is called Peters' rule, which postulates that the probability of synaptic connections can be predicted from the overlap between axonal and dendritic arbours (Rees et al 2017). While overlap between axons and dendrites is necessary for synapses to form, it is far from sufficient. The link between axo-dendritic overlap and connection probability may thus be overly simplistic and requires further scrutiny. While some studies have found support for Peters' rule at the level of functional synaptic connectivity for interneurons (Fino \& Yuste 2011, Packer et al 2013, Rieubland et al 2014), its general applicability has been refuted, at least for local networks, by dense anatomical reconstructions of retinal (Briggman et al 2011, Helmstaedter et al 2013, Kim et al 2014, Krishnaswamy et al 2015) and cortical circuits (Kasthuri et al 2015, Lee et al 2016). To investigate how this principle applies to long-range projections, a technique that has become widely adopted is subcellular channelrhodopsin-assisted circuit mapping (sCRACM). Here optogenetics is combined with spatially targeted optical stimulation to map the distribution of synaptic currents for a given input (Petreanu et al 2009). While this has been used to show that different presynaptic populations target dendritic subdomains with high specificity (Anastasiades et al 2021, Collins et al 2018, Hooks et al 2013, Yamawaki et al 2019), the extent to which this can be explained and predicted by the distribution of axons and dendrites remains largely an open question.

Whether axons target specific dendritic domains is a particularly important question in the case of layer 5 pyramidal neurons (L5PN), given their central role in several theories of cortical computation (Aru et al 2020, Guerguiev et al 2017, Larkum 2013, Richards et al 2019). For example, the interaction between FF and FB information streams across cortical layers (Larkum et al 2018) is thought to underlie sensory 
perception (Larkum 2013, Takahashi et al 2020, Takahashi et al 2016) and implement global inference algorithms such as predictive coding (Shipp 2016). These theories all rely heavily on the assumption that FF connections target primarily basal dendrites while FB connections preferentially synapse onto the apical tuft, and would need to be revised should this not be true. This assumption in turn rests on Peter's rule, but the evidence for this remains circumstantial and a direct examination of Peters' rule across multiple input pathways to individual neurons remains to be done.

Here we present a comprehensive description of the functional input connectivity to thick-tufted layer 5 (ttL5) pyramidal neurons in medial secondary visual cortex. In particular, we set out to answer three questions: determine the source of input connectivity to ttL5 neurons using monosynaptically restricted rabies tracing (Kim et al 2015, Reardon et al 2016), create a census of subcellular input maps using sCRACM, and test Peters' rule directly by comparing synaptic input maps to the respective axonal projection maps.

\section{Results}

To ensure recording from a homogeneous neuronal population, we used the Colgalt2-Cre mouse line which specifically labels subcortically projecting, thick-tufted layer 5 (ttL5) neurons (Groh et al 2010, Kim et al 2015). We focused our study on the medial secondary visual cortex (V2M) as higher order cortical regions are likely to receive a broader diversity of long-range inputs than primary sensory cortices. V2M is defined in the Mouse Brain In Stereotaxic Coordinates atlas (Franklin \& Paxinos 2007) which can be used to guide viral injections. Furthermore, as this atlas is based on cytoarchitecture, thus V2M can be visually distinguished and selectively targeted in slice recordings, as has previously been done (Galloni et al 2020, Young et al 2021). For whole-brain rabies tracing, on the other hand, we adopted the more recently developed Allen Common Coordinate Framework (CCFv3, (Wang et al 2020a). This atlas allowed us to localize individual neurons within 3D volumes of brain tissue, which is not possible using the Franklin \& Paxinos atlas. Within the CCFv3, area V2M corresponds to VISpm, VISam, and RSPagl (Lyamzin \& Benucci 2019), all of which are known to be visually responsive (Garrett et al 2014, Powell et al 2020). Treating V2M as a single area for this study was also supported by the observation that axonal projections to VISpm, VISam, and RSPagl are not substantially different (Figure S1, see Methods for details).

\section{Brain-wide input map to V2M ttL5 pyramidal neurons}

We employed a monosynaptically restricted rabies virus approach (Reardon et al 2016, Wickersham et al 2007 ) to generate a presynaptic input map of V2M ttL5 neurons. Briefly, a mix of adeno-associated viruses carrying floxed N2c G-protein, or TVA-receptor and EGFP genes were injected into V2M of Colgalt2-Cre mice under stereotaxic guidance. Five to seven days later, mCherry expressing EnvA-CVS- 

pipeline (see Methods for details).
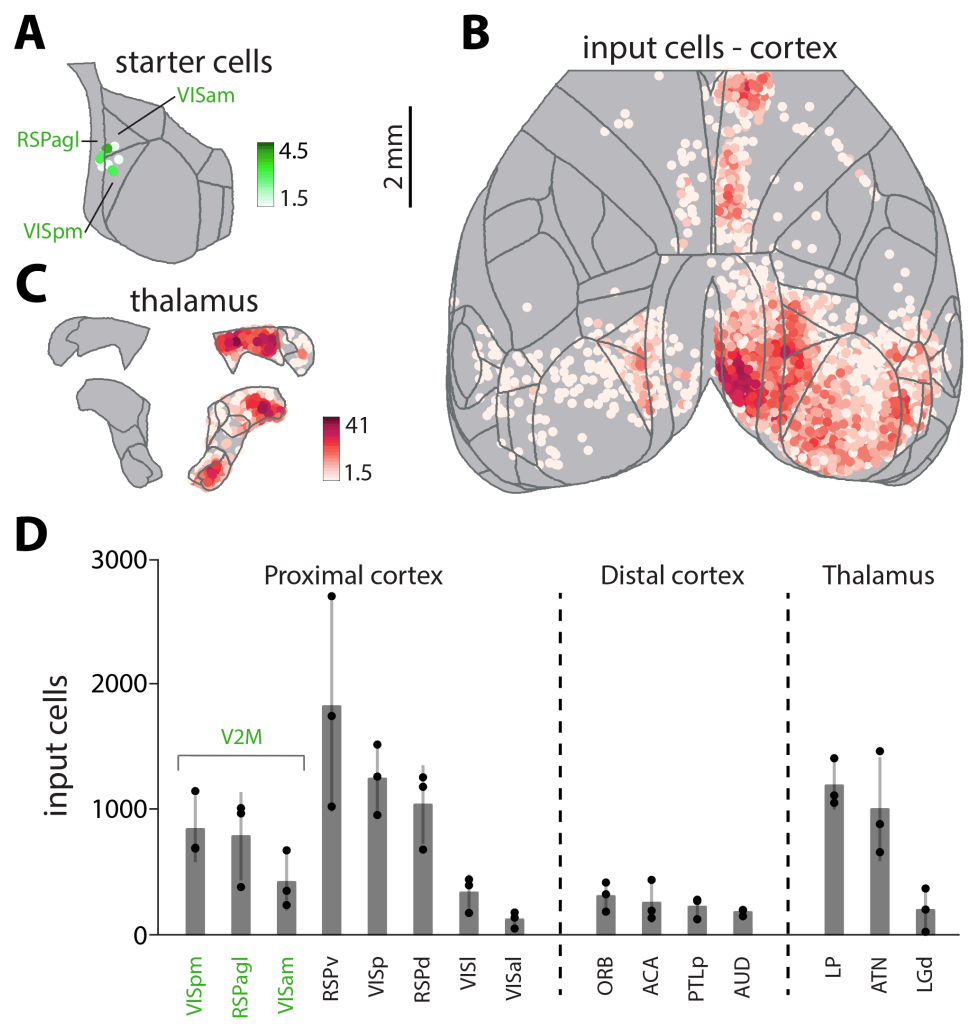

Figure 1. Whole-brain input map to V2M ttL5 neurons. A. Starter cell density map from an example experiment. B. Cortical input cell density map projected onto the horizontal plane, same experiment as in A. C. Thalamic input cell density map projected onto two coronal planes, same experiment as in A. Area names can be found in Figure S3; density scales are in cells / $0.01 \mathrm{~mm}^{2}$. D. Input cell numbers for the most prominent input areas. Averages, standard deviation, and individual experiments are show.

Cell density maps for an example experiment are shown in Figure 1. Starter cells were scattered across V2M (Figure 1A) while presynaptic input neurons were detected in a broad range of cortical and subcortical areas (Figure 1B,C). We have grouped the most prominent input areas into proximal cortex, distal cortex, and thalamus (Figure 1D). The majority of input cells were found locally in V2M and in the proximal cortical areas VISp and the granular retrosplenial cortex (RSPg, consisting of RSPd and RSPV). Orbitofrontal cortex (ORB) and the anterior cingulate area $(A C A)$ provided the most numerous distal cortical inputs. Interestingly, while most cortical input cells were detected in the granular and infragranular layers, especially layer 5, input from ORB was almost exclusively from layer 2/3 (Figure S2BC).

Prominent thalamic inputs were also observed, originating mainly in the lateral posterior nucleus (LP) and 
anterior thalamic nuclei (ATN). Comprehensive cell counts for individual experiments can be found in Supplementary table 1.

112 To understand the organization of inputs onto ttL5 neurons in V2M, we chose to further examine 7 113 prominent input areas. VISp and V2M for FF input; RSPg, ACA and ORB for cortical FB input; and LP 114 and ATN for thalamic FB connections. We designate local (V2M) input as FF, as ttL5 neurons are considered the outputs of the cortical column, and have very limited local projections.

\section{Subcellular optogenetic input mapping reveals diverse targeting of dendritic} 117 domains by input areas

118 To determine the spatial distribution of synaptic inputs to ttL5 neurons in V2M, we performed SCRACM experiments from selected input areas identified by the rabies tracing. Following expression of the optogenetic activator Chronos in different input areas (see methods for injection details), we made voltage-clamp recordings (at $-70 \mathrm{mV}$ ) from tdTomato labelled (Colgalt2-Cre) ttL5 neurons in V2M using acute brain slices. Optical stimulation with a $463 \mathrm{~nm}$ laser was spatially targeted using a digital micromirror device (Figure 2A). Sodium and potassium channels were blocked using TTX $(1 \mu \mathrm{m})$ and 4AP $(100 \mu \mathrm{m})$ to ensure that evoked currents were restricted to directly stimulated nerve terminals and to enhance presynaptic release, respectively. The stimulus consisted of $24 \times 12$ spots of light in a $1000 \times$ $500 \mu \mathrm{m}$ grid aligned to the axis of the apical dendrite of the recorded neuron and covering the whole depth of cortex. We also quantified the total input from a given connection by recording synaptic currents evoked by full-field stimulation. To facilitate comparison between projections, we used the same laser intensity across all experiments.

130 Synaptic strength at each location was estimated by measuring the area of evoked synaptic currents (corresponding to charge; Figure $2 \mathrm{~B}$ ) and creating normalized 2D maps of the spatial distribution of inputs (Figure $2 \mathrm{C}$ ). Individual maps were then aligned (either to the pia or soma) and averaged. To quantify the spatial location of inputs, we projected the average 2D maps in directions parallel (Figure 2C) or perpendicular to the apical dendrite (Figure 2D). Furthermore, we defined the spatial distribution of the three main dendritic compartments based on 11 morphologically reconstructed Colgalt2-Cre neurons (Figure S4). Basal dendrites were defined as those originating at the soma, oblique dendrites as those originating from the apical trunk before the main bifurcation (including the apical trunk itself), and apical tuft dendrites as those originating after the bifurcation. As all three dendritic compartments have similar spine densities (Romand et al 2011), the horizontal projection of the average morphology was used to separate the contribution of each dendritic domain to the total synaptic input (Figure 2D).

141 One potential concern when recording distal synaptic currents from a somatic electrode is the effect of 142 attenuation on detectability of currents. In neurons with weaker overall input, this might result in distal 143 currents becoming too small to detect, thus biasing the input map towards the soma. We tested this by 144 examining the correlation between the location of synaptic input and the total synaptic charge evoked by 

detection bias for distal inputs.
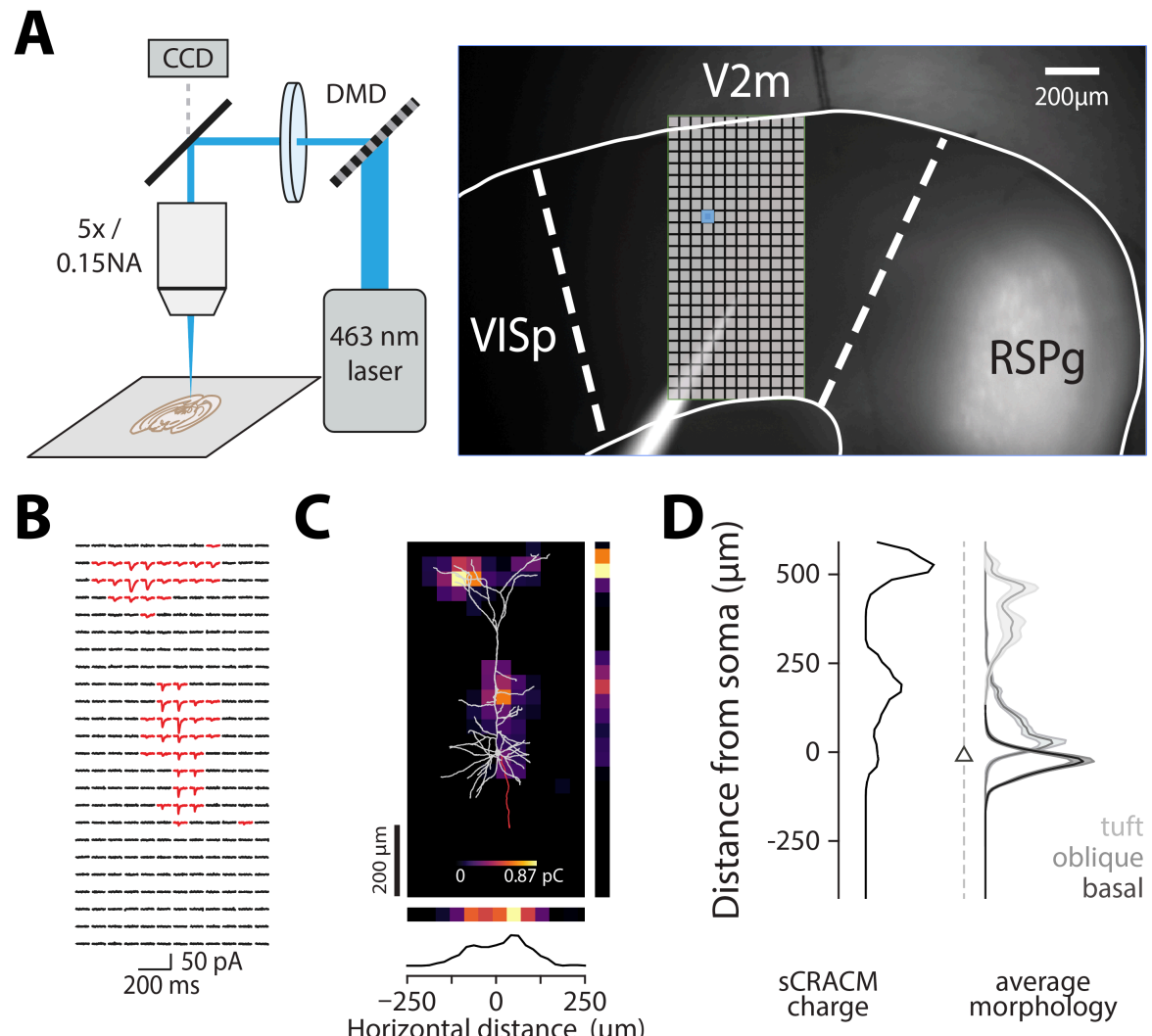

Figure 2. Using SCRACM to map subcellular connectivity. A. Experimental setup and micrograph showing a brain slice with Chronos expression in RSPg and recording pipette in V2M. The stimulation grid is overlaid, and an example spot is highlighted in blue. B. SCRACM recording of excitatory synaptic currents (red $>7 x$ baseline S.D.) from an example neuron. C. Charge heatmap corresponding to recording in B with the morphology of the recorded neuron overlaid. D. Normalized vertical profile of the input map in C. Right: average morphology profile used for dendritic domain deconvolution.

\section{Primary visual cortex}

We first recorded optically evoked synaptic responses arising from VISp axons ( $n=9$ cells from 6 animals, average soma depth $507 \pm 22 \mu \mathrm{m}$; Figure $3 \mathrm{~A}$ ). The apical tuft received $42 \%$ of the input, with a peak input located $188 \mu \mathrm{m}$ from the pia (Supplementary table 2). The remaining input was spread between the oblique compartment, receiving $33 \%$, and basal dendrites, receiving $26 \%$ of the total input. More of the recorded neurons had the peak input in the apical compartment $(n=5 / 9)$ while 4 cells

160 lacked apical input (Figure S6). The horizontal input distribution showed a slight medial skew (towards $161 \mathrm{RSPg})$, most prominent in the oblique $(63 \mu \mathrm{m})$ and basal compartments (42 $\mu \mathrm{m}$; Figure S6B). The total synaptic charge measured via the somatic recording following full-field stimulation was $0.93 \pm 0.11 \mathrm{pC}$ (Figure 3A). VISp thus provides moderate direct input to ttL5 neurons in V2M, primarily targeting the 
$\mathrm{Ai}$

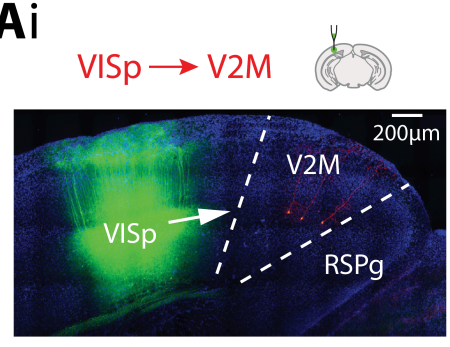

B i

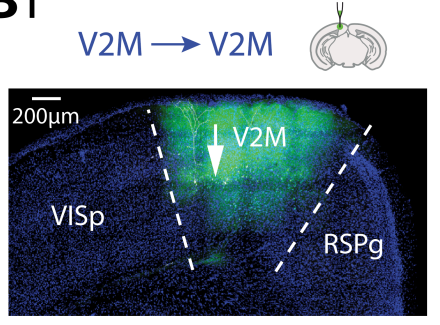

ii

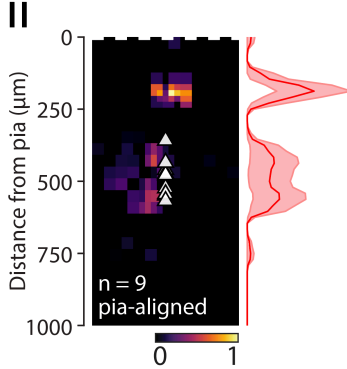

ii

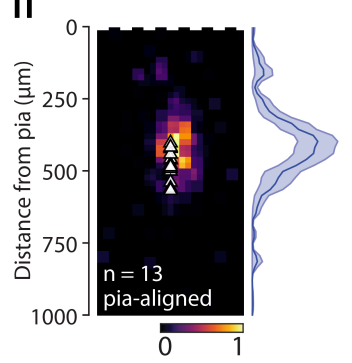

iii

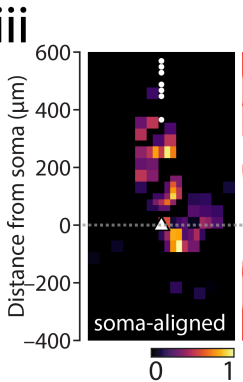

iv

iii

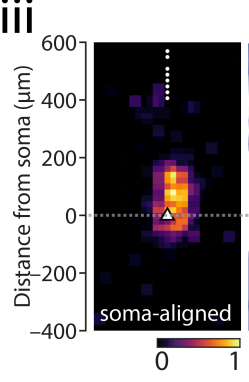

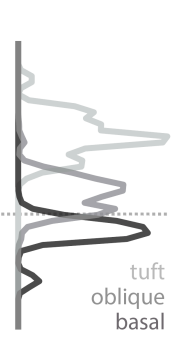

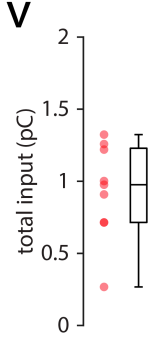

iv

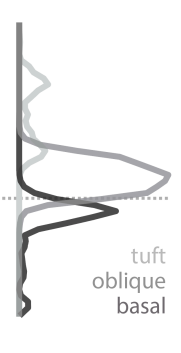

V

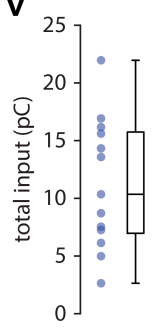

Figure 3. Subcellular connectivity maps of FF input areas. A. $i$ : confocal image of a representative brain slice

(blue = DAPI) showing the injection site in VISp (green) and recorded neurons in V2M (red). ii: pia-aligned average sCRACM heatmap for VISp inputs. Triangles represent soma locations. The vertical profile indicates the normalized average and SEM of the input distributions across all recorded neurons. iii: Same as in ii but aligned on the soma location. Dots indicate pia locations. iv: Normalized input magnitude deconvolved with the average morphology. Dotted line indicates soma location. $v$ : Box plot showing total input charge recorded during full-field stimulation. B. Same as in A but for Cre-off Chronos injections into V2M.

\section{Local input from V2M}

To estimate the distribution of local input we used a Cre-off viral strategy, limiting Chronos expression to non-Colgalt2-Cre neurons ( $n=13$ cells from 4 animals, average soma depth $498 \pm 15 \mu \mathrm{m}$; Figure 3B). When testing this strategy using the much denser Rbp4-Cre line, we found that only a very small proportion of Cre-positive cells expressed Chronos (3\%, Figure S7). The peak input was located close to the soma, at $396 \mu \mathrm{m}$ from the pia. The oblique compartment received the majority (62\%) of this input, with the basal dendrites and apical tuft receiving $24 \%$ and $14 \%$, respectively, of the total input (Supplementary table 2). For the majority of recorded neurons, the peak input occurred perisomatically $(n=12$ / 13; Figure $\mathrm{S6C})$. The horizontal input distribution showed slight medial bias $(-21 \mu \mathrm{m}$ for all peaks; Figure S6D). The total synaptic charge triggered by full-field stimulation was $11.24 \pm 1.56 \mathrm{pC}$ (Figure 3B). Local neurons thus provide large direct input to ttL5 neurons in V2M, primarily targeting the oblique (6.96 pC) compartment, with smaller input arriving to the basal $(2.68 \mathrm{pC})$ and tuft $(1.6 \mathrm{pC})$ compartments. 


\section{Granular retrosplenial area}

187 Next, we recorded optically evoked synaptic responses arising from RSPg axons ( $\mathrm{n}=20$ cells from 9

188

189

190

191

192

193

194

195

animals, average soma depth $503 \pm 15 \mu \mathrm{m}$; Figure 4A). The overall input displayed a bimodal distribution peaking at 125 and $500 \mu \mathrm{m}$ from the pia. The apical tuft received $30 \%$ of the input, with the oblique compartment receiving $40 \%$ and basal dendrites $30 \%$ of the total input (Supplementary table 2). For the majority of recorded neurons, the peak input targeted the perisomatic dendrites ( $n=18$ / 20; Figure S8A). The horizontal input distribution showed slight medial bias (Figure S8B). Total synaptic charge triggered by full-field stimulation was $3.40 \pm 0.51 \mathrm{pC}$ (Figure 4A). RSPg thus provides a relatively moderate direct input to ttL5 neurons in V2M, targeting the oblique (1.36 pC), basal (1.04 pC) and apical tuft (1.01 pC) compartments to similar extent.

\section{Anterior cingulate area}

Next, we recorded optically evoked synaptic responses arising from ACA axons ( $n=23$ cells from 5 animals, average soma depth $464 \pm 9 \mu \mathrm{m}$; Figure 4B). The overall input was bimodal, peaking at $83 \mu \mathrm{m}$ and $438 \mu \mathrm{m}$ from the pia. The apical tuft received $25 \%$ of the input, with the oblique compartment receiving $45 \%$ and basal dendrites $30 \%$ of the total input (Supplementary table 2). The majority of recorded neurons had the peak input located perisomatically ( $n=22$ / 23; Figure S8C). The horizontal input distribution showed no medio-lateral bias (Figure S8D). The total synaptic charge triggered by fullfield stimulation was $9.46 \pm 1.32 \mathrm{pC}$ (Figure 4B). ACA thus provides a large direct input to ttL5 neurons in V2M, primarily targeting the oblique (4.22 pC) compartment with smaller input arriving to the basal (2.83 $\mathrm{pC})$ and most distal part of the apical tuft $(2.41 \mathrm{pC})$.

\section{Orbitofrontal cortex}

Optically evoked synaptic responses arising from ORB axons $(n=11$ cells from 3 animals, average soma depth $521 \pm 19 \mu \mathrm{m}$; Figure 4C) showed a strong perisomatic bias, with a peak at $417 \mu \mathrm{m}$ from the pia. The apical tuft received only $9 \%$ of all input, with the oblique compartment receiving $57 \%$ and basal dendrites $35 \%$ of the total input (Supplementary table 2 ). This distribution was also highly homogeneous across neurons, with almost all recorded neurons having their peak input in the perisomatic region $(\mathrm{n}=$ 11/11; Figure S8E). The horizontal input distribution showed no lateral bias (Figure S8F). The total synaptic charge triggered by full-field stimulation was $7.16 \pm 1.43 \mathrm{pC}$ (Figure $4 \mathrm{C}$ ). ORB thus provides a large direct input to ttL5 neurons in V2M, primarily targeting the oblique (4.05 pC) and basal (2.47 pC) compartments, with slight input arriving to the proximal part of the apical tuft $(0.63 \mathrm{pC})$. 
$\mathbf{A i}$

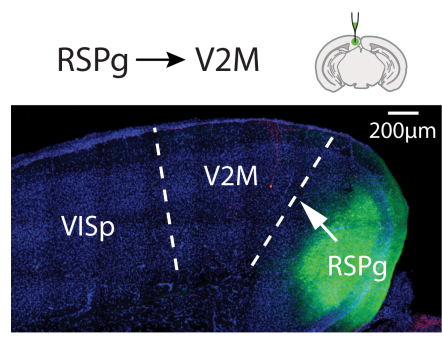

$\mathbf{B i}$

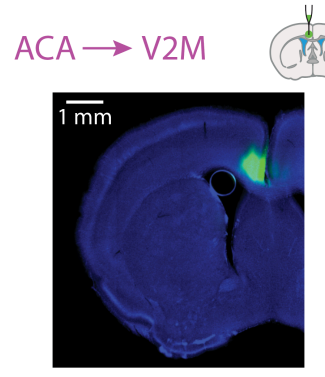

$\mathbf{C i}$

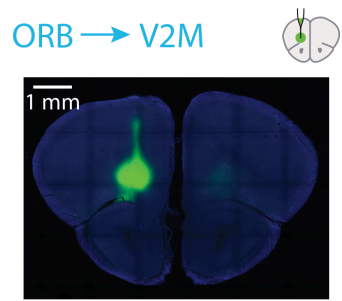

ii

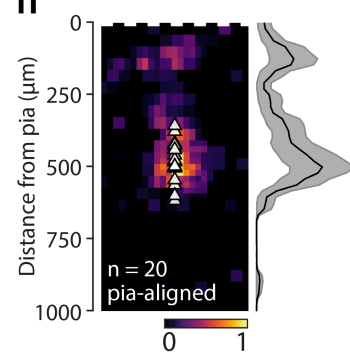

ii

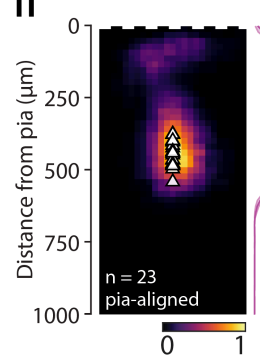

ii
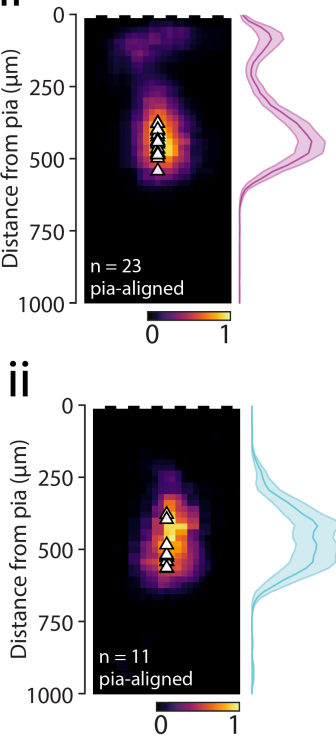

iii
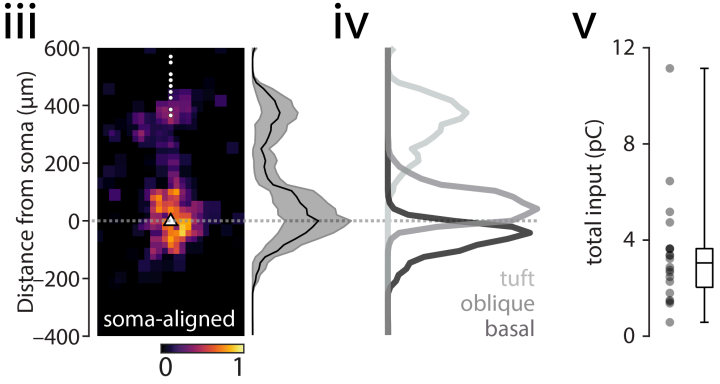

iii

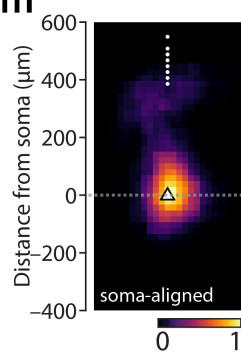

iii

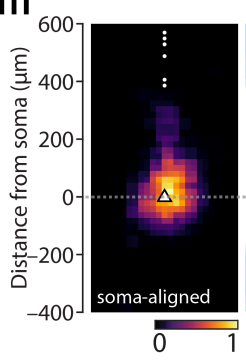

iv
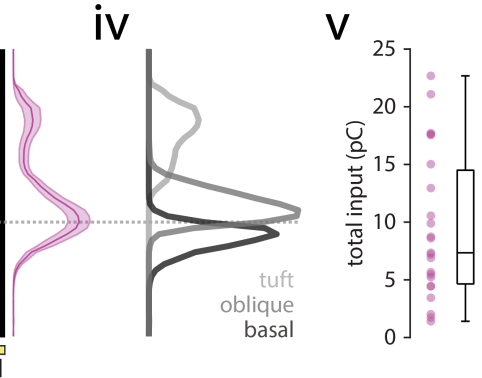

iv

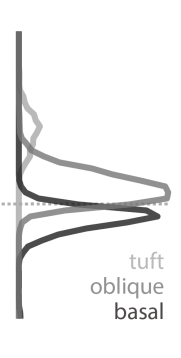

$\mathrm{v}$

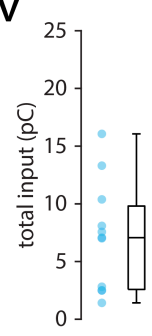

Figure 4. Subcellular connectivity maps of cortical FB areas. A. $i$ : confocal image of a representative brain slice (blue = DAPI) showing the injection site in RSPg (green). ii: pia-aligned average sCRACM heatmap for RSPg inputs.

Triangles represent soma locations. The vertical profile indicates the normalized average and SEM of the input distributions across all recorded neurons. iii: Same as in ii but aligned on the soma location. Dots indicate pia locations. iv: Normalized input magnitude deconvolved with the average morphology. Dotted line indicates soma location. $v$ : Box plot showing total input charge recorded during full-field stimulation.

B. Same as in A but for Chronos injections into ACA. C. Same as in A but for Chronos injections into ORB.

\section{Anterior thalamic nuclei}

Next, we recorded optically evoked synaptic responses from thalamic axons, starting with the ATN ( $n=8$ cells from 3 animals, average soma depth $435 \pm 15 \mu \mathrm{m}$; Figure 5A). This input had peaks at both $104 \mu \mathrm{m}$ and $333 \mu \mathrm{m}$ from the pia, with the apical tuft receiving the majority $(75 \%)$ of the input, while the oblique compartment received $17 \%$ and basal dendrites a mere $8 \%$ of the total input (Supplementary table 2).

230 The majority of recorded neurons had the peak input in the tuft compartment $(n=6 / 8)$ and while all cells

231 had some tuft input, in 2/8 cells the input peak was located perisomatically (Figure S9A). The horizontal input distribution showed a medial bias (Figure S9B). The total synaptic charge triggered by full-field 
Ai

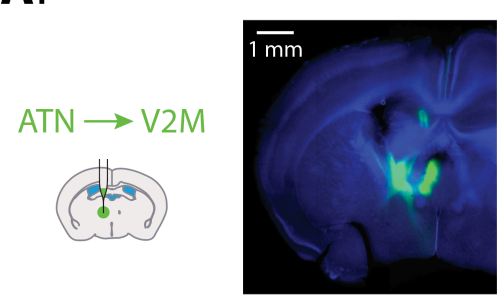

Bi

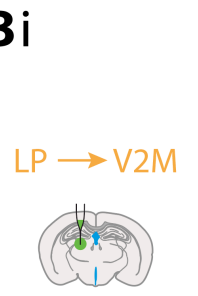

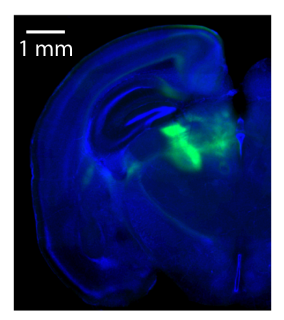

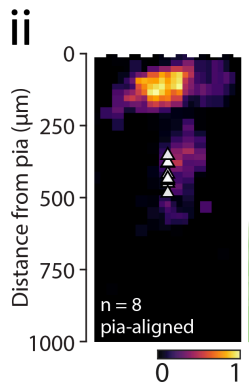

ii

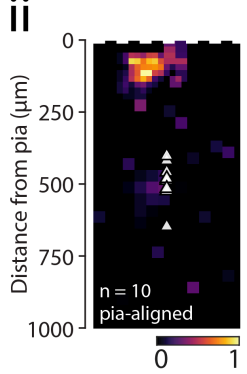

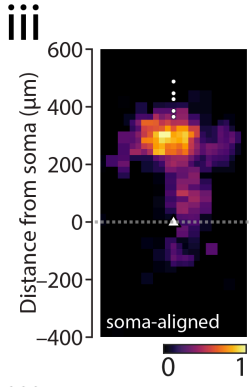
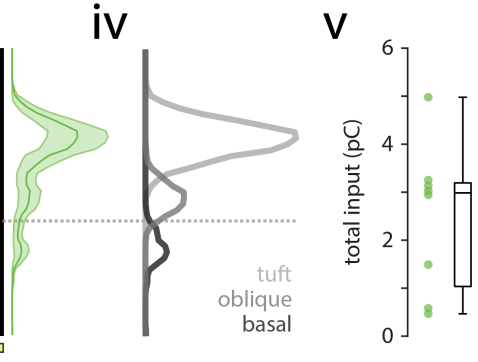

iii

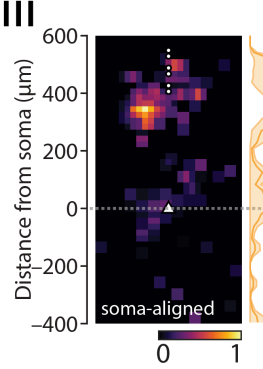

iv

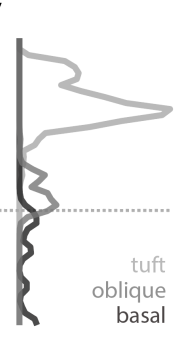

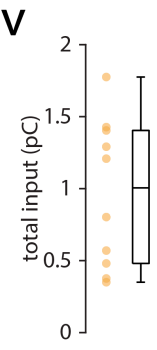

Figure 5. Subcellular connectivity maps of thalamic input areas. A. $i$ : confocal image of a representative brain slice (blue = DAPI) showing the injection site in ATN (green). ii: pia-aligned average sCRACM heatmap for ATN inputs. Triangles represent soma locations. The vertical profile indicates the normalized average and SEM of the input distributions across all recorded neurons. iii: Same as in ii but aligned on the soma location. Dots indicate pia locations. iv: Normalized input magnitude deconvolved with the average morphology. Dotted line indicates soma location. $v$ : Box plot showing total input charge recorded during full-field stimulation.

B. Same as in A but for Chronos injections in LP.

\section{Lateral posterior nucleus of the thalamus}

Lastly, we recorded optically evoked synaptic responses arising from LP axons ( $n=10$ cells from 4 animals, average soma depth $500 \pm 23 \mu \mathrm{m}$; Figure $5 \mathrm{~B}$ ). Due to excessive retrograde labelling resulting in direct photocurrent in the recorded V2M cells, a 1:10 dilution of virus was used for these injections and the absolute value of the evoked input is thus likely an underestimate. As with ATN axons, the LP input was strongly biased towards the most superficial part of the cortex and peaked at $63 \mu \mathrm{m}$ from the pia. The apical tuft received the vast majority (75\%) of the input, with the oblique compartment receiving $15 \%$ and basal dendrites $10 \%$ of the total input (Supplementary table 2). Most recorded neurons had the peak input in the tuft compartment ( $n=9 / 10$; Figure S9C). The horizontal input distribution showed lateral bias

254 (Figure S9D). The total synaptic charge triggered by full-field stimulation was $0.97 \pm 0.16 \mathrm{pC}$ (Figure 5B).

255 LP thus provides modest direct input to ttL5 neurons in V2M, primarily targeting the most distal part of the 
apical tuft $(0.72 \mathrm{pC})$ compartment with smaller input arriving to the oblique $(0.14 \mathrm{pC})$ and the basal $(0.10$

pC) compartments.

\section{Comparison of anatomical and functional connectivity maps}

Having determined the spatial distribution of synapses for the main input areas, we next sought to directly compare this to what would be predicted from axo-dendritic overlap (i.e. from Peters' rule). To determine axonal projection patterns from input areas to V2M, we have imaged the Chronos-eGFP labelled axons in a subset of the brain slices used for the SCRACM experiments using confocal microscopy.

A $\mathrm{VISp} \rightarrow \mathrm{V} 2 \mathrm{M} \quad \mathrm{ORB} \rightarrow \mathrm{V} 2 \mathrm{M}$
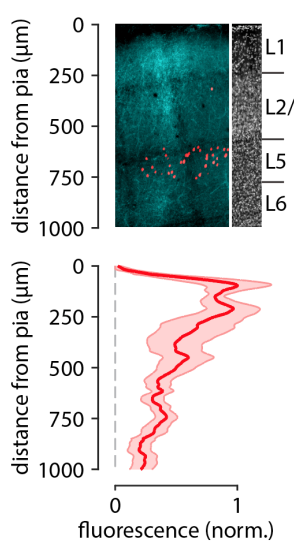

B
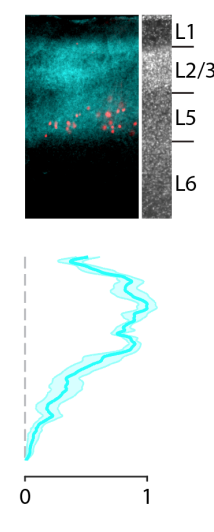

$\mathrm{RSPg} \rightarrow \mathrm{V} 2 \mathrm{M} \quad \mathrm{ACA} \rightarrow \mathrm{V} 2 \mathrm{M}$
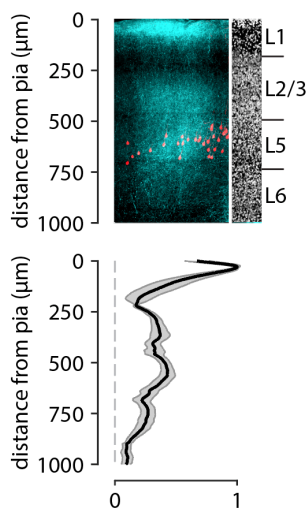
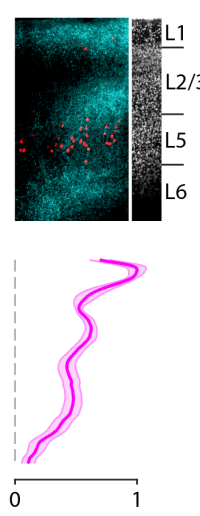

C

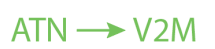
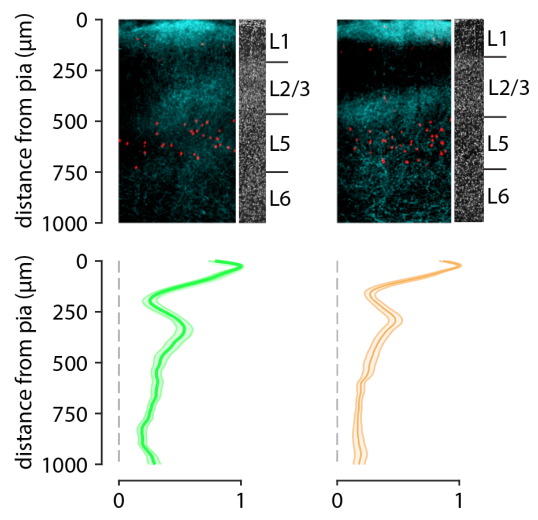

Figure 6. Axonal projection densities from different input areas. A-C. Top: example confocal images from V2M showing axonal projections from six input areas (cyan) and Colgalt2-Cre cell bodies (red). The corresponding DAPI staining shows variation in laminar depth. Bottom: average projection density profiles across the cortical depth averaged across 3-5 injections.

The spatial distribution of axons followed three basic patterns. Axons from VISp and ORB were densest in layer (L) 2/3 and L5 while little projection was apparent in L1, reminiscent of the classical FF projection pattern (Figure 6A). In contrast, axons from RSPg and ACA showed an FB-like pattern with dense labelling in the middle part of $L 1$ followed by sparse labelling in $L 2$ and diffuse axons in layers 3,5 and 6 (Figure 6B). The final group, which consists of the thalamic projections from LP and ATN, showed the classical FB pattern strongly innervating the external part of $L 1$, with a secondary peak in $L 3$, but little or no projections in layers 2, 5 and 6 (Figure 6C).

To accurately estimate morphological overlap between axons and dendrites, we multiplied the axonal

277 projection maps with the average dendritic morphology, resulting in the predicted input distribution one

278 would expect to see based on Peters' rule. When overlaying this with the pia-aligned vertical SCRACM

279 maps, the alignments between functional synapses and the axo-dendritic maps were diverse (Figure 7A). 
280 For some regions, like ORB perisomatic and LP tuft inputs, a clear correspondence could be seen

281 between predicted and measured input distributions. A lesser degree of overlap can be seen in the VISp

282 perisomatic or ACA tuft inputs. For other inputs, however, strong functional input could be detected where

283 there is little overlap between dendrites and axons, such as at VISp tuft inputs. This stood in stark

284 contrast to the ORB projection, for which the opposite was true, and apical regions of dense

285 morphological overlap of axons and dendrites resulted in no functional input.

286 Next we examined the correspondence between the anatomical input connectivity obtained from rabies

287 tracing and the functional connectivity measured by total synaptic input. The number of rabies-labelled

288 input neurons showed a strong contribution from RSPg and V2M, and modest input cell numbers for the

289 more distal cortical regions (e.g. ACA, ORB). The total synaptic input, however, shows no correlation with

290 these numbers $(p=0.8, r=-0.14$, Spearman correlation, Figure 7B), with modest synaptic input from

291 RSPg and most input arriving from V2M, ACA and ORB. Taken together, these results show clear

292 specificity of dendritic targeting by brain-wide connections, with only a loose adherence to Peters' rule for

293 most inputs as well as large differences between anatomical and functional connectivity measured by

294 rabies tracing and optogenetic stimulation, respectively. 
A
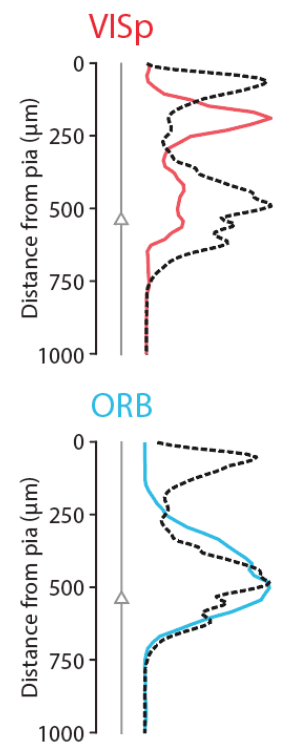

B

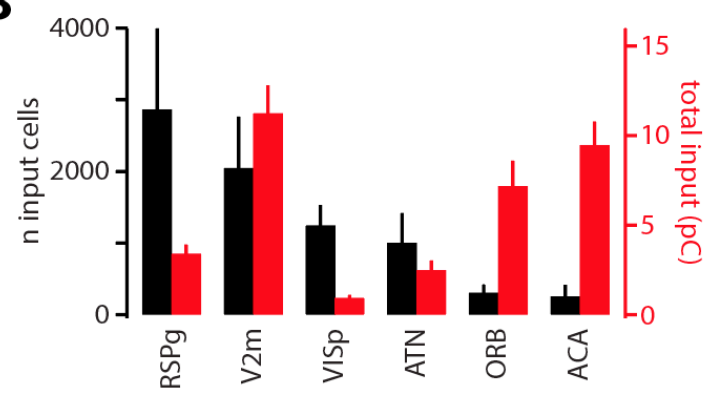

C
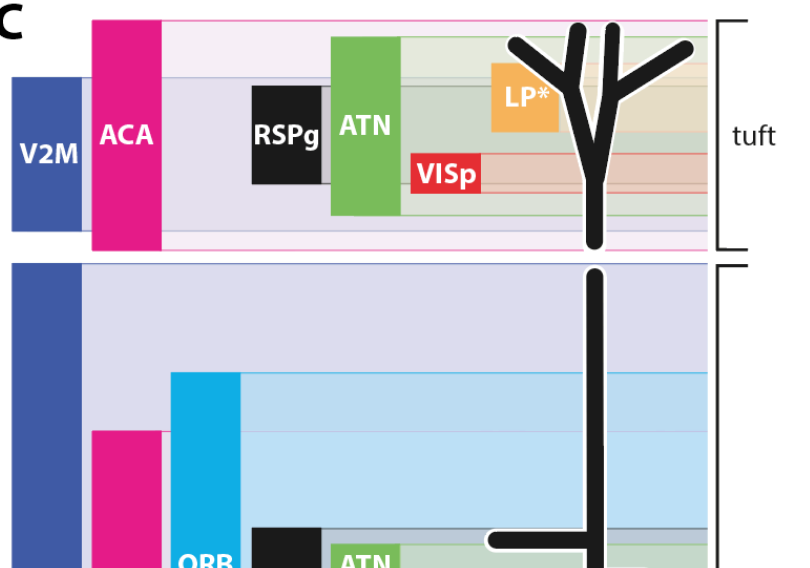

V2M

ORB

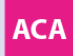
RSPg ATN ACA VISp
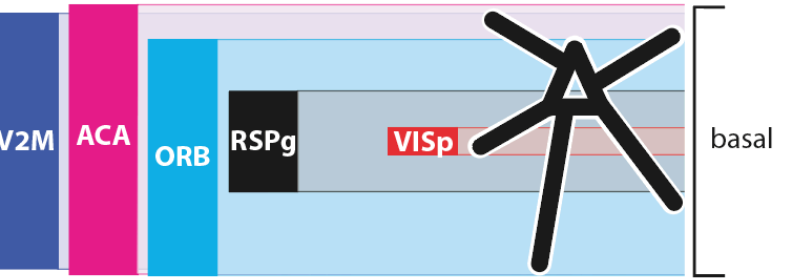

Figure 7. Comparison of different input maps. A. Axonal density distributions multiplied with dendritic morphology (dotted black lines) overlaid with pia-aligned synaptic input distributions (coloured lines). Six input areas are shown. Triangles represent soma location in average morphology. B. Number of input cells across 6 areas established by rabies tracing (black) and total input charge recorded during full-field optogenetic stimulation (red). C. Schematic of excitatory synaptic input map to ttL 5 pyramidal neurons. The height of bars represents input strength, while the centre of each bar is aligned to the peak of the SCRACM map. The tuft input map was generated from pia aligned maps while oblique and basal maps from soma aligned maps. NB: LP input magnitude $\left(^{*}\right)$ is likely underestimated due to 


\section{Discussion}

307 Using an array of techniques for long-range circuit dissection, we have comprehensively mapped the

308 location and dendritic targets of inputs to ttL5 neurons in the medial secondary visual cortex in mice. This

309 allowed us to determine the dendritic targets of FF and FB connections and to make a direct assessment

310 of Peters' rule for brain-wide connections.

311 The whole-brain input map generated via rabies tracing was qualitatively similar to previous results from

312 the primary visual cortex (Kim et al 2015). Axonal projections from the rabies-identified input regions

313 broadly followed the expected pattern, with FB projections being biased toward L1 and FF areas toward

314 the deeper layers (D'Souza et al 2020, Harris et al 2019, Rockland \& Pandya 1979). Accordingly, L1 was

315 densely innervated by higher-order cortical areas like RSPg and ACA as well as the secondary thalamic

316 nuclei (LP, ATN). Interestingly, ORB, a higher-order cortical region, displayed a projection pattern

317 associated with FF areas. Additionally, the majority of ORB projection neurons were found in L2/3,

318 another feature of FF connectivity. ORB thus seems to be an exception in terms of FB axonal projections.

319 Compared to the axonal projection patterns, synaptic input maps showed a remarkable degree of

320 heterogeneity. Morphological averaging was necessary as the SCRACM recordings in our dataset did not

321 have paired reconstructions for every cell. While it is possible that comparing the individual axon,

322 dendrite, and synaptic profiles on a single-cell basis would have given slightly more accurate results, the overall pattern of functional input from each input region was mostly consistent across cells. Morphologies of ttL5 neurons are likewise highly stereotypical. Furthermore, the axon projection patterns used for evaluating Peters' rule were measured from a subset of the same slices used for the SCRACM recordings, further supporting the direct comparison of the predicted input maps with those recorded functionally. The discrepancy resulting from averaging is thus likely to be low. Indeed, any smoothing resulting from averaging of morphologies, axonal projections or SCRACM maps would only increase overlap, and thus bias the results in favor of adhering to Peters' rule. In contrast to this, several connections showed only weak correspondence between predicted and observed input maps. The connection from VISp, which is FF by definition and as such is assumed to primarily target perisomatic dendrites (Larkum 2013), was instead biased towards the apical tuft. Conversely, while the axons from ORB had a FF-like projection pattern, they synapsed almost exclusively with basal and oblique dendrites. Other recorded areas (e.g., RSPg, ACA, ATN) showed some degree of conformity to Peters' rule, yet still with significant differences in the proportion of synapses generated in the different regions of high axonal projections. The only area where the correspondence was remarkable and Peter's rule held fully was LP.

337 Comparing anatomical connectivity obtained by rabies tracing to functional connectivity obtained by full338 field optogenetic stimulation of axons revealed large and unexpected differences. When evaluating this finding, it is important to consider a few technical caveats which might bias this comparison. First, while 
341 provided input), there is considerable debate regarding the quantitative accuracy of rabies tracing

342 (Rogers \& Beier 2021). Second, the magnitude of optogenetically evoked input depends on the number of

343 presynaptic cells covered by viral injection. To facilitate comparison with the rabies labelling, we aimed to

344 maximise coverage of each area by making several injections targeted to the locations with the highest

345 density in the rabies data. It is unlikely that these technical caveats could alone account for the

346 remarkable discrepancy between anatomical and functional input magnitudes. There are several other

347 possible explanations for this difference. First, there may be differing convergence of connectivity

348 between input areas. For example, low convergence in inputs with strong rabies labelling, with a unique

349 mapping of selected pre- and postsynaptic cells, could result in relatively weaker sCRACM input (like

350 VISp and RSPg). Meanwhile, strong synaptic currents relative to small rabies-labelled populations (like

351 ORB and ACA) may be explained by higher convergence. Such connections might be less discerning of

352 their targets in order to convey more general contextual or state-specific information. Second, there may

353 be a difference in the strength of individual synapses not reflected in rabies efficiency, with sparsely

354 labelled input areas like ORB having relatively strong synapses, while rabies-dense areas like RSPg may

355 send a large number of weaker synapses. A third contributing factor could be the recently reported

356 activity-dependence of rabies transmission (Beier et al 2017). The apparent sparsity of some input areas

357 (like ORB and ACA) could thus arise from having very low activity. Conversely, to result in extensive

358 rabies labelling, VISp and RSPg should provide high activity input.

359 We used a novel approach to allocate sCRACM input to specific dendritic compartments by deconvolving

360 the synaptic input maps using morphological ttL5 reconstructions in which we manually labelled tuft,

361 oblique, and basal compartments. Our results provide a complex picture regarding the possible

362 interaction of FF and FB inputs (Figure 7C). Functionally, V2M has been linked to visual motion

363 processing (Sun et al 2009) and is thought to take part in navigation and spatial processing as part of the

364 dorsal stream (Glickfeld \& Olsen 2017). Thalamic FB input, which targets almost exclusively the apical

365 tuft, arrives from multiple higher-order nuclei. Parts of ATN receive strong vestibular input (Rancz et al

3662015 ) and, together with RSP form a central part of the head-direction system (Taube 2007, Velez-Fort et

367 al 2018). It is thus likely that spatial and multisensory contextual information carried by LP inputs (Roth et

368 al 2016) interacts with FF visual input in the tuft compartment. However, the role of tuft integration is likely

369 to differ from primary sensory cortices, considering that ttL5 neurons in the secondary visual cortex have

370 substantially different integrative properties (Galloni et al 2020). Contrary to thalamic input, cortical FB

371 inputs target all three dendritic domains. Perhaps surprisingly, the strongest of these are ORB and ACA,

372 which can interact with the local FF input at the level of oblique and basal dendrites. Frontal cortices in

373 general are involved in decision making and executive control of behavior (Hamilton \& Brigman 2015),

374 and ORB in particular has been shown to encode spatial goals (Feierstein et al 2006). ACA, meanwhile,

375 can directly regulate visually evoked responses and sensory discrimination in the primary visual cortex

376 (Zhang et al 2014), and contributes to learning to predict sensory input to primary visual cortex (Fiser et al

377 2016). Their precise roles in the functioning of V2M, however, remains unknown. The strong input 
378 received by oblique dendrites is particularly important, as this compartment was shown to strongly affect

379 L5 excitability (Schaefer et al 2003) and can gate information flow from the apical dendrites (Jarsky et al

380 2005). While FB inputs targeting the apical tuft have been suggested to act as a general gain control for

381 ttL5 neurons, it is thus possible that ORB and ACA perform a similar gating function, but perhaps with

382 more specificity regarding input identity or dendritic branches. Indeed, their particularly strong targeting of

383 oblique dendrites might allow both fine-level control of plasticity for FF synapses at these dendrites, while

384 simultaneously allowing them to exert gating control over input from both the thalamic nuclei and VISp,

385 which strongly project to the tuft.

386 In general, our results show that while the classification of areas as FF or FB can be based on axonal

387 projections (albeit with exceptions, such as ORB), macroscopic projectomes do not predict cell-type level input location, and individual connections do not follow clear rules associated with their position above or

389 below the target area in a cortical hierarchy. Similarly, while rabies tracing from a population of starter

390 cells is an effective tool to study the general wiring diagram, the proportion of input neurons thus

391 estimated is likely to give a poor estimation of functional input strength. Finally, the location and possible

392 interactions between FF and the broad range of FB inputs as well as their specific information content

393 suggests that ttL5 neurons may be adopting a multitude of integrative strategies that are more complex

394 than those previously suggested. 


\section{Materials and Methods}

\section{Animals}

All animal experiments were prospectively approved by the local ethics panel of the Francis Crick Institute (previously National Institute for Medical Research) and the UK Home Office under the Animals (Scientific Procedures) Act 1986 (PPL: 70/8935). All surgery was performed under isoflurane anesthesia, and every effort was made to minimize suffering. Transgenic mice were used: $\mathrm{Tg}$ (Colgalt2-Cre)NF107Gsat (RRID:MMRRC_036504-UCD, also known as GIt25d2-Cre) were crossed with the Ai14 reported line expressing tdTomato (RRID:IMSR_JAX:007908). Additionally, Tg(Rbp4-Cre)KL100Gsat/Mmucd (RRID:MMRRC_031125-UCD) mice were used to establish the efficacy of the cre-off approach. As only male mice are transgenic in the Colgalt2-Cre line, all experiments were done on male animals. Animals were housed in individually ventilated cages under a $12 \mathrm{hr}$ light/dark cycle.

\section{Viruses}

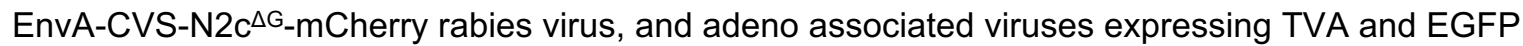
(AAV8-EF1a-flex-GT), N2c glycoprotein (AAV1-Syn-flex-H2B-N2CG), or Cre-OFF Chronos-GFP (AAV1EF1-CreOff-Chronos-GFP) were a generous gift of Molly Strom and Troy Margrie. Chronos-GFP (also called ShChR) expressing adeno associated virus (rAAV1-Syn-Chronos-GFP) was obtained from UNC Vector Core.

\section{Surgical procedures}

Surgeries were performed on mice aged 3-8 weeks using aseptic technique under isoflurane (2-4\%) anesthesia and analgesia (meloxicam $2 \mathrm{mg} / \mathrm{kg}$ and buprenorphine $0.1 \mathrm{mg} / \mathrm{kg}$ ). The animals were headfixed in a stereotaxic frame and a small hole $(0.5-0.7 \mathrm{~mm})$ was drilled in the skull above the injection site. Virus was loaded into a glass microinjection pipette (pulled to a tip diameter of around $20 \mu \mathrm{m}$ ) and pressure injected into the target region at a rate of $0.4 \mathrm{nl} / \mathrm{s}$ using a Nanoject III delivery system (Drummond Scientific). To reduce backflow, the pipette was left in the brain for approximately 5 min after completion of each injection.

For rabies virus tracing experiments, a 1:2 mixture of TVA and N2c glycoprotein expressing credependent AAVs (10-20 nL) was injected at stereotaxic brain coordinates $(\lambda-0.8 \mathrm{~mm}, \mathrm{ML} 1.6 \mathrm{~mm}$, DV 0.6 mm). Rabies virus (50-100 $\mathrm{nL})$ was injected 5-7 days later at the same site. Ten to twelve days later, animals were transcardially perfused under terminal anesthesia with cold phosphate-buffer (PB, $0.1 \mathrm{M})$ followed by $4 \%$ paraformaldehyde (PFA) in PB (0.1 M).

For the sCRACM experiments, Chronos-GFP expressing AAV was injected into one of the identified presynaptic regions. The virus was allowed to express for at least 3 weeks before acute brain slice 
preparation. The range of stereotaxic coordinates for each region are listed in Supplementary table 3. The injected virus was Chronos-GFP for every region except V2M, where Cre-OFF Chronos-GFP was instead used to avoid expression in the recorded Colgalt2-Cre neurons. For some of the injections in LP, the Chronos-GFP virus was diluted by 10 -fold in sterile cortex buffer before injection.

\section{Data acquisition and analysis for rabies tracing experiments}

Brain samples were embedded in 4-5\% agarose (Sigma-Aldrich: 9012-36-6) in 0.1M PB and imaged using serial two-photon tomography (Han et al 2018, Osten \& Margrie 2013, Ragan et al 2012). Eight optical sections were imaged every $5 \mu \mathrm{m}$ with $1.2 \mu \mathrm{m} \times 1.2 \mu \mathrm{m}$ lateral resolution, after which a $40 \mu \mathrm{m}$ physical section was removed. Excitation was provided by a pulsed femto-second laser at $800 \mathrm{~nm}$ wavelength (MaiTai eHP, Spectraphysics). Images were acquired through a 16X, 0.8 NA objective (Nikon MRP07220) in three channels (green, red, blue) using photomultiplier tubes. Image tiles for each channel and optical plane were stitched together with an open-source software written in MATLAB (https://github.com/SainsburyWellcomeCentre/Stitchlt). For cell detection, full resolution images were first filtered with a Gaussian blur (sigma $=1$ ) using Fiji (ImageJ 1.52e) to reduce imaging noise. The opensource package "cellfinder" (Tyson et al 2020) was used for cell candidate detection then classification. Automated mouse atlas propagation (Niedworok et al 2016) was used for registration and segmentation on brain samples down-sampled to $10 \mu \mathrm{m}$ voxels (to match the resolution of the Allen CCFv3; (Wang et al 2020a). Cell coordinates were similarly down-sampled to $10 \mu \mathrm{m}$ and the number of cells was counted for each segmented area. For cell density visualisation, cell coordinates were reverse-transformed onto the Allen CCFv3 space using the open source registration tool, Elastix (Klein et al 2010) and were projected onto a $2 \mathrm{D}$ matrix in $10 \mu \mathrm{m} /$ pixel resolution.

\section{Acute slice preparation and electrophysiological recordings}

Adult mice were deeply anaesthetised with isoflurane and decapitated. The brain was rapidly removed and placed in oxygenated ice-cold slicing solution containing (in $\mathrm{mM}$ ): 125 sucrose, $62.5 \mathrm{NaCl}, 2.5 \mathrm{KCl}$, $1.25 \mathrm{NaH}_{2} \mathrm{PO}_{4}, 26 \mathrm{NaHCO}_{3}, 2 \mathrm{MgCl}_{2}, 1 \mathrm{CaCl}_{2}, 25$ dextrose; osmolarity 340-350 mOsm. The cerebellum and frontal cortex were removed manually with a coronal cut using a single-edged razor blade and the rostral surface was affixed to a metal platform with cyanoacrylate glue. Coronal slices (300 $\mu \mathrm{m}$ thick) between 2.6 and $3.5 \mathrm{~mm}$ posterior to bregma were prepared using a vibrating blade microtome (Leica VT1200S). Slices were kept submerged in artificial cerebrospinal fluid (ACSF, containing in mM: 125 $\mathrm{NaCl}, 2.5 \mathrm{KCl}, 1.25 \mathrm{NaH}_{2} \mathrm{PO}_{4}, 26 \mathrm{NaHCO}_{3}, 1 \mathrm{MgCl}_{2}, 2 \mathrm{CaCl}_{2}$, 25 dextrose; osmolarity 308-312 mOsm) at $35^{\circ} \mathrm{C}$ for the first $30-60 \mathrm{~min}$ after slicing, then at room temperature $\left(22^{\circ} \mathrm{C}\right)$. All solutions and chambers were continuously bubbled with carbogen (95\% O2 / 5\% CO2).

The topology of the cortical mantle in the region of V2m is curved and varies in thickness along the antero-posterior axis. In most coronal sections, the apical dendrites of $L 5$ neurons were thus at a slight 
angle relative to the slicing surface. This angle was minimized by slicing the brain with a slight backward angle relative to the coronal plane. Additionally, to avoid recording from neurons with cut apical dendrites, slices were placed such that the apical dendrites could be seen to descend at a shallow angle into the slice. Where possible, the fluorescently-labelled apical dendrites were also visually inspected along their full path from soma to pia. Furthermore, many neurons were successfully filled with biocytin during recording, making it possible to verify the integrity of the apical dendrite after the recordings. The observed neurons were all found to have an intact apical trunk with tuft dendrites extending to the pia. However, we can't exclude that a small fraction of finer dendrites (including both basal and apical tuft) extending towards the slice surface may have been partially cut in the process.

For recordings, individual slices were perfused in the recording chamber at a rate of approximately 6 $\mathrm{mL} / \mathrm{min}$ with ACSF at room temperature $\left(22^{\circ} \mathrm{C}\right)$, continuously bubbled with carbogen. To prevent axonal spike propagation and enhance responses to optical stimulation, $1 \mu \mathrm{M}$ tetrodotoxin (TTX) and $100 \mu \mathrm{M} 4$ aminopyridin (4-AP) were added to the recording ACSF. This ensured that any light-evoked responses were direct monosynaptic responses resulting from stimulation of Chronos-expressing axon terminals, rather than from passing axons terminating in unknown locations on the dendrites.

Filamented borosilicate thick-walled glass micropipettes were pulled and heat-polished using a two-stage horizontal puller (Zeitz DMZ Universal Electrode Puller) to obtain an electrode resistance of 3-6 M $\Omega$. The glass electrodes were filled with internal solution optimized for voltage clamp recordings, containing (in mM): $120 \mathrm{CsMeSO}_{3}\left(\mathrm{CH}_{3} \mathrm{O}_{3} \mathrm{SCs}\right), 3 \mathrm{CsCl}, 10 \mathrm{HEPES}, 1$ EGTA, 4 Na2ATP, $0.3 \mathrm{NaGTP}, 5$ Na2phosphoreatine $\left(\mathrm{C}_{4} \mathrm{H}_{8} \mathrm{~N}_{3} \mathrm{O}_{5} \mathrm{PNa}_{2}\right)$, $3.5 \mathrm{QX}-314$ chloride, $0.5 \%$ (w/v) biocytin hydrochloride, $50 \mu \mathrm{M}$ Alexa Fluor 488 hydrazide; osmolarity 290-295 mOsm; pH adjusted to 7.3 with $\mathrm{CsOH}$.

Visually guided whole-cell patch-clamp recordings from tdTomato-labelled Colgalt2-Cre neurons in V2M were performed using a Scientifica SliceScope Pro 3000 microscope equipped with a 40x/0.8 NA objective and an infrared (IR) Dodt Gradient Contrast system. The epifluorescence system used to visualize fluorescent neurons was a CoolLED pE-4000 illumination system with a $550 \mathrm{~nm}$ peak excitation wavelength. To avoid stimulating Chronos-expressing axons, epifluorescent illumination was kept to a minimum during selection of cells to record. Recordings were made with a Multiclamp 700B amplifier (Molecular Devices) in voltage-clamp configuration with a holding potential of $-70 \mathrm{mV}$. Filtered signals (8kHz low-pass) were digitized at $20 \mathrm{kHz}$ with a National Instruments DAQ board (PCle-6323). Acquisition and stimulus protocols were generated in Igor Pro (Wavemetrics) with the NeuroMatic software package (Rothman \& Silver 2018). Throughout each recording, series resistance compensation was applied and set to the highest value possible without inducing oscillations in the cell (typically between 40 and $75 \%$ ). Recordings wtih series resistance larger than $40 \mathrm{M} \Omega$ were excluded. 


\section{Patterned optogenetic stimulation}

495 Optical stimulation was implemented using a digital micromirror device (DMD) with a $463 \mathrm{~nm}$ laser (lasercoupled Polygon 400, Mightex Systems). The stimulus consisted of a 1000 x $500 \mu \mathrm{m}$ grid divided into 24 $x 12$ spots of light $(41.7 \mu \mathrm{m} \times 41.7 \mu \mathrm{m}$ square) delivered through a 5x/0.15 NA dry objective (Olympus MPlanFL N). The grid was approximately centered on the soma being recorded from, aligned to the pia orthogonal to the apical dendrite. For each individual spot, the laser power was measured at the specimen plane using a PM100D (Thorlabs) optical power meter equipped with a S121C sensor (Thorlabs). The laser output associated with each spot was adjusted to obtain a measured power of approximately $300 \mu \mathrm{W}(173 \mathrm{~mW} / \mathrm{mm} 2)$.

Optical stimuli were delivered for $1 \mathrm{~ms}$ at $0.1 \mathrm{~Hz}$ in a pseudo-random sequence designed to maximise the distance between consecutive spots and the time between stimulation of neighbouring spots. Each recording trial consisted of a single repetition of all 288 stimuli followed by a full-field stimulus, in which all stimulation spots were illuminated simultaneously for $1 \mathrm{~ms}$. For each cell, 5-20 trials were recorded, with 30s pauses between trials, making the interval between consecutive stimulation of the same spot approximately 60s. Following each recording, an image was taken to record the location of the recorded cell (filled with Alexa Fluor 488) relative to the stimulation grid. This was used during analysis to align the recorded SCRACM heatmap with the location of the pia or soma.

\section{Immunohistochemistry \& morphological reconstructions}

After recording, slices were fixed overnight at $4^{\circ} \mathrm{C}$ in a $4 \%$ paraformaldehyde solution and were subsequently kept in PBS. Slices were stained with DAPI $(5 \mu \mathrm{g} / \mathrm{mL})$ for $10 \mathrm{~min}$, mounted on glass slides and images were acquired with either a confocal microscope for high-resolution images (Leica SP5; objective: 20x/0.7NA or 10x/0.4NA; pinhole size: 1 airy unit) or a slide scanner for visualizing injection sites (Olympus VS120, objective: 4x/0.16NA). Image processing was done with the FIJI software package (Schindelin et al 2012). For the detailed morphological analysis, a subset of neurons, selected based on the quality and completeness of staining, was reconstructed in full through the LMtrace service of https://ariadne.ai//mtrace.

\section{Comparison of axonal projection patterns to VISam, VISpm and RSPagl}

We have obtained layer-wise axonal projection data from the 6 input areas (ACA $n=33$, ATN $n=11$, LP $n=10$, ORB $n=11, R S P g n=17, V I S p n=60)$ from the Allen Mouse Brain Connectivity database (@ 2011 Allen Institute for Brain Science. Allen Mouse Brain Connectivity Atlas. Available from: https://connectivity.brain-map.org/). Projection energy distributions were qualitatively similar across target areas (Figure S1). Quantitatively, the data followed the same layer-wise pattern across the three target areas ( $p>0.05$, 2-way ANOVA with Tukey's post hoc test) with the exception of ACA (RSPagl vs VISpm $p=0.001$; VISam vs VISpm $p=0.016$ ). When only wild-type data was considered, no statistical 
difference between target areas was detected ( $p>0.05$, 2-way ANOVA with Tukey's post hoc test; $n=$ ACA 5, ATN 4, LP 2, ORB 2, RSPg 2, VISp 21).

531 Analysis and data visualization were performed with custom macros and scripts written in Igor Pro and

532 MATLAB (Mathworks). Unless otherwise specified, all reported data values refer to the mean \pm standard

533 error (SEM). Recordings were not corrected for liquid junction potential.

534 Recordings were baselined in a $40 \mathrm{~ms}$ window before each stimulus and averaged across trials, and the

535 peak and area of the evoked currents were measured in a $50 \mathrm{~ms}$ window after the stimulus. Stimulus

536 spots for which the peak current was lower than seven times the standard deviation of the baseline noise

537 were scored as zero.

538 For some of the injection sites, a degree of retrograde transport of the Chronos virus was noted in areas

539 outside of the primary injection site, including in V2M. A few recorded Colgalt2-Cre neurons in V2M were

540 thus found to be intrinsically expressing Chronos. This was easily detected in the recordings by an

541 instantaneous inward current at the onset of laser stimulation, in contrast to the 4-5 ms delay between

542 stimulus onset and SCRACM current observed normally. Any cells with no such delay were excluded from

543 the analysis.

544 Because peak response amplitudes varied between cells and preparations, to obtain average input

545 distributions from a presynaptic population, the heatmap for each cell was normalised to the peak EPSC

546 value for that cell. Heatmaps were then aligned horizontally by soma location and then vertically by either

547 soma or pia location before averaging each pixel across cells. For each cell, the soma could be localised

548 to within one quadrant of a given stimulation spot. When averaging across cells, the effective sampling

549 resolution (i.e. the pixel dimension) for the average SCRACM heatmaps and related horizontal and

550 vertical projections were thus approximately $20.8 \mu \mathrm{m}$ (equal to half of the stimulus spot size, i.e. 1000/48

$551 \mu \mathrm{m})$. All values reported for the locations of sCRACM inputs from different presynaptic regions are in

552 multiples of this number. Note, however, that the actual resolution with which synapses can be localised

553 in the heatmaps is likely to be lower than this, as it is limited by both light scattering in the tissue and by

554 the spread of voltage along stimulated axons, which is determined by the length constant of presynaptic

555 axons. Previous studies have indicated that these factors limit the actual SCRACM resolution to

556 approximately $60 \mu \mathrm{m}$ (Petreanu et al 2009).

557 To estimate the proportion of SCRACM input targeting different dendritic domains, the recorded input map

558 for each cell was convolved with the average ttL5 morphology obtained from 11 reconstructed Colgalt2-

559 Cre neurons in V2M. This was done by manually separating the apical tuft, oblique (including the apical

560 trunk), and basal dendrites of the reconstructions in Neurolucida 360 and quantifying the total dendritic

561 length in $10 \mu \mathrm{m}$ thick sections perpendicular to the main axis of the apical dendrite. The resulting dendrite

562 profiles were then aligned by the soma and averaged. Using this average morphology, at each distance 
from the soma the proportion of dendrites belonging to each domain was calculated relative to the total dendritic length within that section. For each SCRACM recording, these proportions were calculated using an average morphology that was scaled to the soma-pia distance of the recorded cell. Each pixel of the heatmap for that cell was then multiplied by these dendritic proportions to obtain the proportion of evoked current assigned to each dendritic domain. Averaging the morphological reconstructions was necessary because only a small fraction of the recorded neurons was fully reconstructed, and we were thus unable to allocate SCRACM measures to specific dendritic domains on a cell-by-cell basis. Notably, because of variation in apical dendrite length between different ttL5 neurons, the choice of soma alignment before averaging the morphologies resulted in the average morphology profile having a graded rather than sharp cutoff at the pia. The peak dendrite density near the pia thus appears smaller than it would in a piaaligned average and is not related to any loss of dendrites in the reconstructed neurons. This choice was made because the distribution of tuft dendrites had relatively little overlap with the other dendritic domains, resulting in a more accurate assignment of SCRACM inputs to each domain. Had we aligned the morphologies by the pia instead, the resulting distributions for basal and oblique dendrites would have far greater overlap, resulting in lower accuracy in the dendritic domain classification.

578 The average morphology profile was also used to quantify the expected input profile on the basis of axon

579 and dendrite densities at each distance from the pia. In this case, however, the same reconstructed

580 morphologies were instead aligned by the apical tuft before averaging, in order to make the predicted

581 input more comparable to the pia-aligned SCRACM maps. As with the sCRACM input, pia-alignment resulted in greater definition at the pia at the cost of reduced resolution near the average soma location.

583 Following alignment, both the axon and dendrite distributions were normalized to the peak of each curve

584 and multiplied, resulting in large values for expected input at locations containing both axons and 585 dendrites.

\section{Acknowledgments}

588 We thank Troy Margrie and Molly Strom for viral constructs; Rob Campbell and Charlie Rousseau for help 589 with data acquisition and analysis of rabies tracing experiments, and Joe Brock for help with illustrations.

590 We are grateful to Florencia lacaruso and Zoltán Kisvárday for helpful comments on the manuscript.

591 Competing interests: The authors declare that no competing interests exist. 


\section{References}

594

595

596

597

598

599

600

601

602

603

604

605

606

607

608

609

610

611

612

613

614

615

616

617

618

619

620

621

622

623

624

625

626

627

628

629

630

631

632

633

634

635

636

637

638

639

640

641

642

643

644

645

646

Anastasiades PG, Collins DP, Carter AG. 2021. Mediodorsal and Ventromedial Thalamus Engage Distinct L1 Circuits in the Prefrontal Cortex. Neuron 109: 314-30 e4

Aru J, Suzuki M, Larkum ME. 2020. Cellular Mechanisms of Conscious Processing. Trends Cogn Sci 24: 814-25

Beier KT, Kim CK, Hoerbelt P, Hung LW, Heifets BD, et al. 2017. Rabies screen reveals GPe control of cocaine-triggered plasticity. Nature 549: 345-50

Briggman KL, Helmstaedter M, Denk W. 2011. Wiring specificity in the direction-selectivity circuit of the retina. Nature 471: 183-8

Collins DP, Anastasiades PG, Marlin JJ, Carter AG. 2018. Reciprocal Circuits Linking the Prefrontal Cortex with Dorsal and Ventral Thalamic Nuclei. Neuron 98: 366-79 e4

D'Souza RD, Meier AM, Bista P, Wang Q, Burkhalter A. 2016. Recruitment of inhibition and excitation across mouse visual cortex depends on the hierarchy of interconnecting areas. Elife 5

D'Souza RD, Wang Q, Ji W, Meier AM, Kennedy H, et al. 2020. Canonical and noncanonical features of the mouse visual cortical hierarchy. biorxiv.org

Feierstein CE, Quirk MC, Uchida N, Sosulski DL, Mainen ZF. 2006. Representation of spatial goals in rat orbitofrontal cortex. Neuron 51: 495-507

Felleman DJ, Van Essen DC. 1991. Distributed hierarchical processing in the primate cerebral cortex. Cereb Cortex 1: 1-47

Fino E, Yuste R. 2011. Dense inhibitory connectivity in neocortex. Neuron 69: 1188-203

Fiser A, Mahringer D, Oyibo HK, Petersen AV, Leinweber M, Keller GB. 2016. Experience-dependent spatial expectations in mouse visual cortex. Nat Neurosci 19: 1658-64

Franklin KBJ, Paxinos G. 2007. Paxinos and Franklin's The mouse brain in stereotaxic coordinates. Amsterdam: Academic Press, an imprint of Elsevier. 1 volume (unpaged) pp.

Galloni AR, Laffere A, Rancz E. 2020. Apical length governs computational diversity of layer 5 pyramidal neurons. Elife 9

Garrett ME, Nauhaus I, Marshel JH, Callaway EM. 2014. Topography and areal organization of mouse visual cortex. J Neurosci 34: 12587-600

Glickfeld LL, Olsen SR. 2017. Higher-Order Areas of the Mouse Visual Cortex. Annu Rev Vis Sci 3: 25173

Groh A, Meyer HS, Schmidt EF, Heintz N, Sakmann B, Krieger P. 2010. Cell-type specific properties of pyramidal neurons in neocortex underlying a layout that is modifiable depending on the cortical area. Cereb Cortex 20: 826-36

Guerguiev J, Lillicrap TP, Richards BA. 2017. Towards deep learning with segregated dendrites. Elife 6

Hamilton DA, Brigman JL. 2015. Behavioral flexibility in rats and mice: contributions of distinct frontocortical regions. Genes Brain Behav 14: 4-21

Han Y, Kebschull JM, Campbell RAA, Cowan D, Imhof F, et al. 2018. The logic of single-cell projections from visual cortex. Nature 556: 51-56

Harris JA, Mihalas S, Hirokawa KE, Whitesell JD, Choi H, et al. 2019. Hierarchical organization of cortical and thalamic connectivity. Nature 575: 195-202

Helmstaedter M, Briggman KL, Turaga SC, Jain V, Seung HS, Denk W. 2013. Connectomic reconstruction of the inner plexiform layer in the mouse retina. Nature 500: 168-74

Hooks BM, Mao T, Gutnisky DA, Yamawaki N, Svoboda K, Shepherd GM. 2013. Organization of cortical and thalamic input to pyramidal neurons in mouse motor cortex. $J$ Neurosci 33: 748-60

Hubel DH, Wiesel TN. 1962. Receptive fields, binocular interaction and functional architecture in the cat's visual cortex. J Physiol 160: 106-54

Jarsky T, Roxin A, Kath WL, Spruston N. 2005. Conditional dendritic spike propagation following distal synaptic activation of hippocampal CA1 pyramidal neurons. Nat Neurosci 8: 1667-76

Kasthuri N, Hayworth KJ, Berger DR, Schalek RL, Conchello JA, et al. 2015. Saturated Reconstruction of a Volume of Neocortex. Cell 162: 648-61

Kim EJ, Juavinett AL, Kyubwa EM, Jacobs MW, Callaway EM. 2015. Three Types of Cortical Layer 5 Neurons That Differ in Brain-wide Connectivity and Function. Neuron 88: 1253-67

Kim JS, Greene MJ, Zlateski A, Lee K, Richardson M, et al. 2014. Space-time wiring specificity supports direction selectivity in the retina. Nature 509: 331-36 
647

648

649

650

651

652

653

654

655

656

657

658

659

660

661

662

663

664

665

666

667

668

669

670

671

672

673

674

675

676

677

678

679

680

681

682

683

684

685

686

687

688

689

690

691

692

693

694

695

696

697

698

699

700

701

702

Klein S, Staring M, Murphy K, Viergever MA, Pluim JP. 2010. elastix: a toolbox for intensity-based medical image registration. IEEE Trans Med Imaging 29: 196-205

Krishnaswamy A, Yamagata M, Duan X, Hong YK, Sanes JR. 2015. Sidekick 2 directs formation of a retinal circuit that detects differential motion. Nature 524: 466-70

Larkum M. 2013. A cellular mechanism for cortical associations: an organizing principle for the cerebral cortex. Trends Neurosci 36: 141-51

Larkum ME, Petro LS, Sachdev RNS, Muckli L. 2018. A Perspective on Cortical Layering and LayerSpanning Neuronal Elements. Front Neuroanat 12: 56

LeCun Y, Bengio Y, Hinton G. 2015. Deep learning. Nature 521: 436-44

Lee WC, Bonin V, Reed M, Graham BJ, Hood G, et al. 2016. Anatomy and function of an excitatory network in the visual cortex. Nature 532: 370-4

Lyamzin D, Benucci A. 2019. The mouse posterior parietal cortex: Anatomy and functions. Neurosci Res 140: 14-22

Niedworok CJ, Brown AP, Jorge Cardoso M, Osten P, Ourselin S, et al. 2016. aMAP is a validated pipeline for registration and segmentation of high-resolution mouse brain data. Nat Commun 7: 11879

Osten P, Margrie TW. 2013. Mapping brain circuitry with a light microscope. Nat Methods 10: 515-23

Packer AM, McConnell DJ, Fino E, Yuste R. 2013. Axo-dendritic overlap and laminar projection can explain interneuron connectivity to pyramidal cells. Cereb Cortex 23: 2790-802

Petreanu L, Mao T, Sternson SM, Svoboda K. 2009. The subcellular organization of neocortical excitatory connections. Nature 457: 1142-5

Powell A, Connelly WM, Vasalauskaite A, Nelson AJD, Vann SD, et al. 2020. Stable Encoding of Visual Cues in the Mouse Retrosplenial Cortex. Cereb Cortex 30: 4424-37

Ragan T, Kadiri LR, Venkataraju KU, Bahlmann K, Sutin J, et al. 2012. Serial two-photon tomography for automated ex vivo mouse brain imaging. Nat Methods 9: 255-8

Rancz EA, Moya J, Drawitsch F, Brichta AM, Canals S, Margrie TW. 2015. Widespread vestibular activation of the rodent cortex. J Neurosci 35: 5926-34

Reardon TR, Murray AJ, Turi GF, Wirblich C, Croce KR, et al. 2016. Rabies Virus CVS-N2c(DeltaG) Strain Enhances Retrograde Synaptic Transfer and Neuronal Viability. Neuron 89: 711-24

Rees CL, Moradi K, Ascoli GA. 2017. Weighing the Evidence in Peters' Rule: Does Neuronal Morphology Predict Connectivity? Trends Neurosci 40: 63-71

Richards BA, Lillicrap TP, Beaudoin P, Bengio Y, Bogacz R, et al. 2019. A deep learning framework for neuroscience. Nat Neurosci 22: 1761-70

Rieubland S, Roth A, Hausser M. 2014. Structured connectivity in cerebellar inhibitory networks. Neuron 81: $913-29$

Rockland KS, Pandya DN. 1979. Laminar origins and terminations of cortical connections of the occipital lobe in the rhesus monkey. Brain Res 179: 3-20

Rogers A, Beier KT. 2021. Can transsynaptic viral strategies be used to reveal functional aspects of neural circuitry? J Neurosci Methods 348: 109005

Romand S, Wang Y, Toledo-Rodriguez M, Markram H. 2011. Morphological development of thick-tufted layer $\mathrm{v}$ pyramidal cells in the rat somatosensory cortex. Front Neuroanat 5: 5

Roth MM, Dahmen JC, Muir DR, Imhof F, Martini FJ, Hofer SB. 2016. Thalamic nuclei convey diverse contextual information to layer 1 of visual cortex. Nat Neurosci 19: 299-307

Rothman JS, Silver RA. 2018. NeuroMatic: An Integrated Open-Source Software Toolkit for Acquisition, Analysis and Simulation of Electrophysiological Data. Front Neuroinform 12: 14

Schaefer AT, Larkum ME, Sakmann B, Roth A. 2003. Coincidence Detection in Pyramidal Neurons Is Tuned by Their Dendritic Branching Pattern. Journal of Neurophysiology 89: 3143-54

Schindelin J, Arganda-Carreras I, Frise E, Kaynig V, Longair M, et al. 2012. Fiji: an open-source platform for biological-image analysis. Nat Methods 9: 676-82

Shipp S. 2016. Neural Elements for Predictive Coding. Front Psychol 7: 1792

Sun B, Lv B, Dong Q, Wang S, Chai Z. 2009. Watching moving images specifically promotes development of medial area of secondary visual cortex in rat. Dev Neurobiol 69: 558-67

Takahashi N, Ebner C, Sigl-Glockner J, Moberg S, Nierwetberg S, Larkum ME. 2020. Active dendritic currents gate descending cortical outputs in perception. Nat Neurosci 23: 1277-85

Takahashi N, Oertner TG, Hegemann P, Larkum ME. 2016. Active cortical dendrites modulate perception. Science 354: 1587-90 
Taube JS. 2007. The head direction signal: origins and sensory-motor integration. Annu Rev Neurosci 30: $181-207$

705

Tyson AL, Rousseau CV, Niedworok CJ, Keshavarzi S, Tsitoura C, Margrie TW. 2020.

706

707

Velez-Fort M, Bracey EF, Keshavarzi S, Rousseau CV, Cossell L, et al. 2018. A Circuit for Integration of Head- and Visual-Motion Signals in Layer 6 of Mouse Primary Visual Cortex. Neuron 98: 179-91 e6 Framework: A 3D Reference Atlas. Cell 181: 936-53 e20

Wang XJ, Pereira U, Rosa MG, Kennedy H. 2020b. Brain connectomes come of age. Curr Opin Neurobiol 65: 152-61

Wickersham IR, Lyon DC, Barnard RJ, Mori T, Finke S, et al. 2007. Monosynaptic restriction of transsynaptic tracing from single, genetically targeted neurons. Neuron 53: 639-47

Yamawaki N, Li X, Lambot L, Ren LY, Radulovic J, Shepherd GMG. 2019. Long-range inhibitory intersection of a retrosplenial thalamocortical circuit by apical tuft-targeting CA1 neurons. Nat Neurosci 22: 618-26

Young H, Belbut B, Baeta M, Petreanu L. 2021. Laminar-specific cortico-cortical loops in mouse visual cortex. Elife 10

Zeng H. 2018. Mesoscale connectomics. Curr Opin Neurobiol 50: 154-62

Zhang S, Xu M, Kamigaki T, Hoang Do JP, Chang WC, et al. 2014. Selective attention. Long-range and local circuits for top-down modulation of visual cortex processing. Science 345: 660-5 


\section{Supplementary material}

725

726
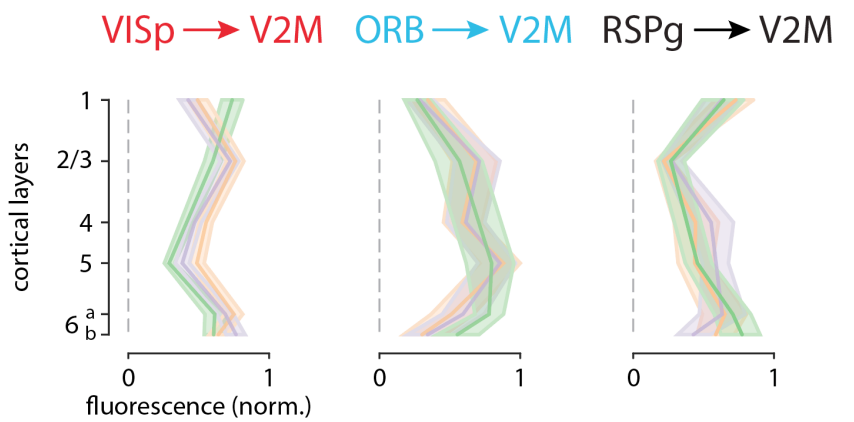

$\mathrm{ACA} \rightarrow \mathrm{V} 2 \mathrm{M}$

$\mathrm{ATN} \rightarrow \mathrm{V} 2 \mathrm{M}$

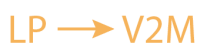

727 Figure S1. Comparison of axonal projection profiles. Layer-wise axonal projection profiles to VISpm, VISam and
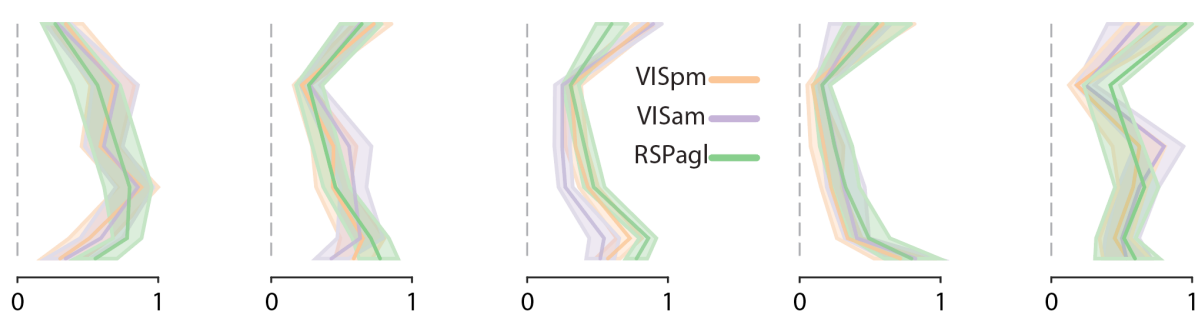

728 RSPagl. Data from the Allen Mouse Brain Connectivity Atlas. 


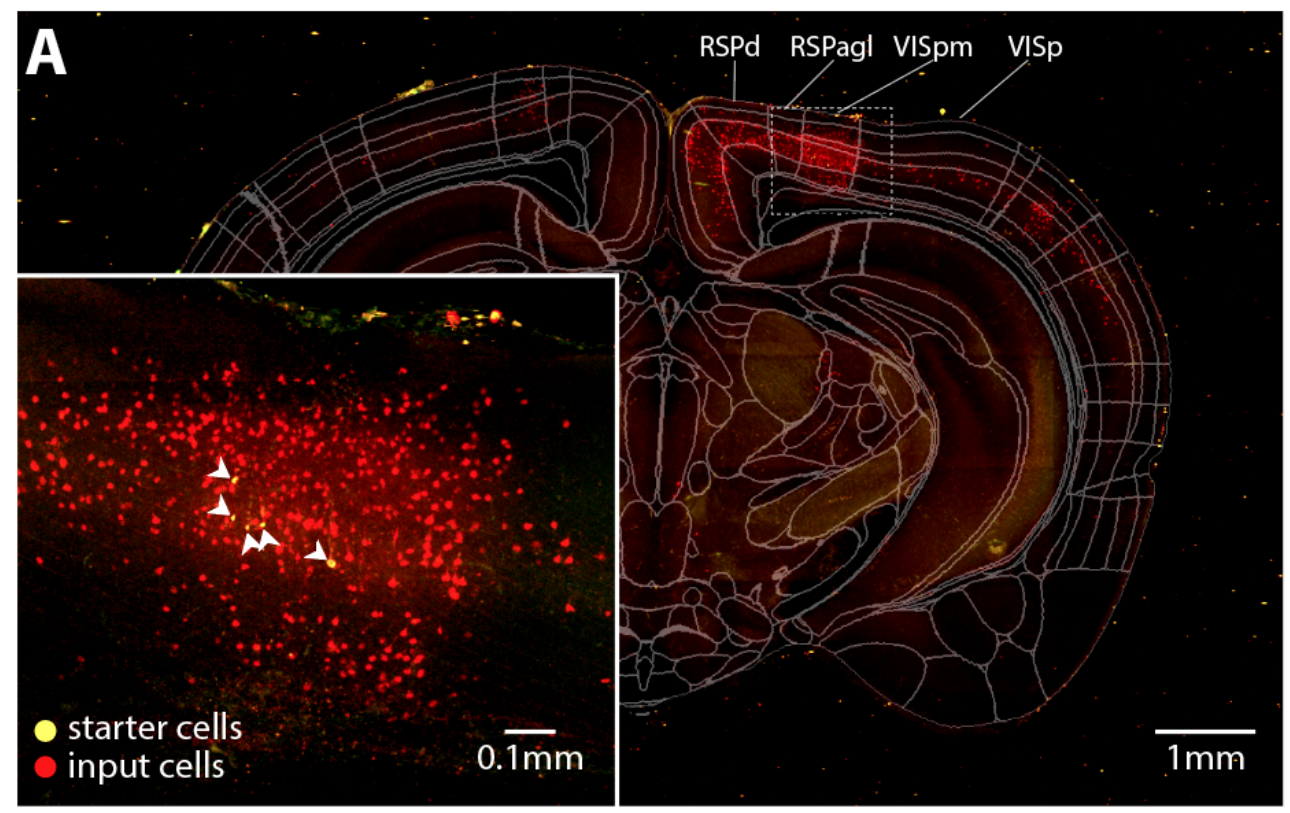

\section{B supragranular}
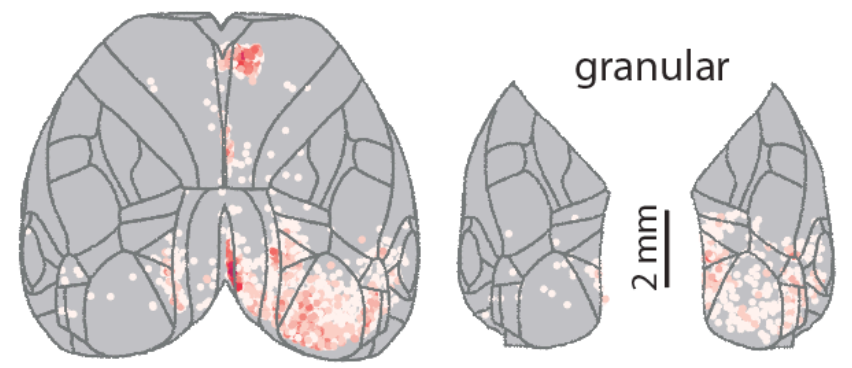

infragranular

C

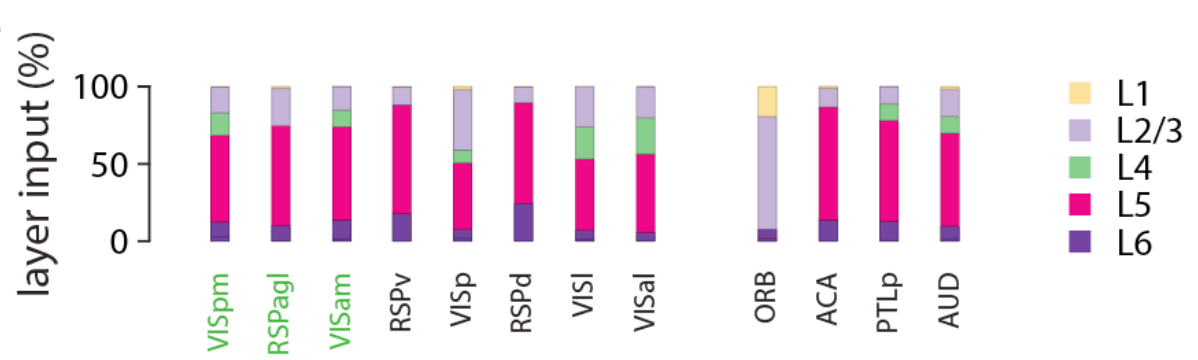

Figure S2. A. Maximum intensity projection of a $200 \mu \mathrm{m}$ thick coronal slab containing some of the starter neurons. The propagated CCFv3 atlas outlines are overlayed. Inset shows starter area with higher magnification. B. Input cell density maps across supragranular, granular and infragranular layers. A-B Same experiment and scales as Figure 1. C. Distribution of input cells across cortical layers. Average of 3 experiments. 


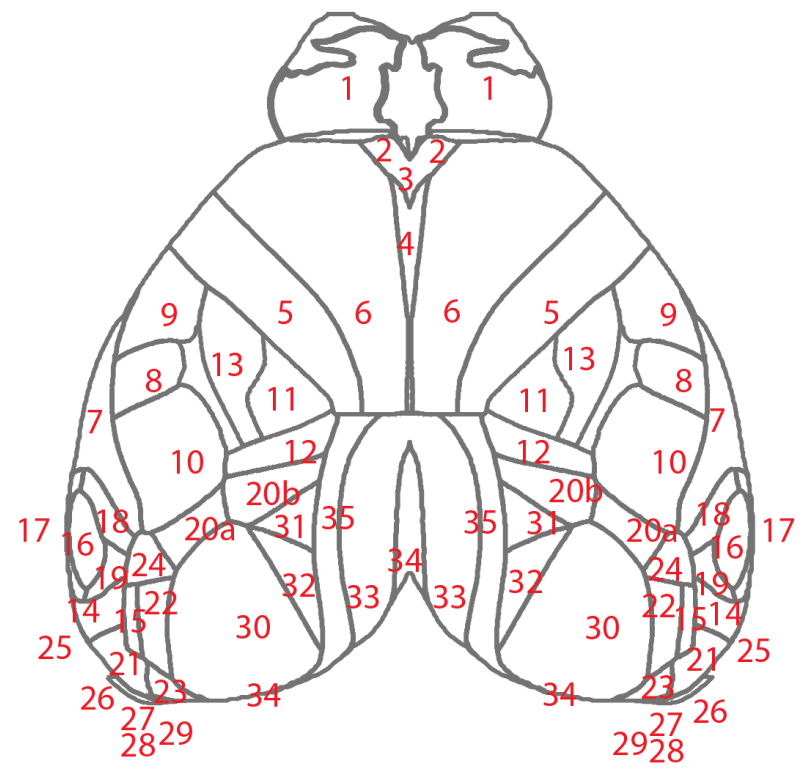

\section{Cortex}

Main olfactory bulb

Orbital area

Prelimbic area

Anterior cingulate area

Primary motor area

Secondary motor area

Supplemental somatosensory area

Primary somatosensory area, nose

Primary somatosensory area, mouth

Primary somatosensory area, barrel field

Primary somatosensory area, lower limb

Primary somatosensory area, trunk

Primary somatosensory area, upper limb

Temporal association areas

Laterointermediate area

Primary auditory area

Ventral auditory area

Dorsal auditory area

Posterior auditory area

\section{Thalamus}

36

37

\begin{abstract}
Lateral posterior nucleus of the thalamus Dorsal part of the lateral geniculate complex Lateral dorsal nucleus of thalamus
\end{abstract}

$\begin{array}{ll}\text { MOB } & 20 \\ \text { ORB } & 20 a \\ \text { PL } & 20 b \\ \text { ACA } & 21 \\ \text { MOp } & 22 \\ \text { MOs } & 23 \\ \text { SSs } & 24 \\ \text { SSp-n } & 25 \\ \text { SSp-m } & 26 \\ \text { SSp-bfd } & 27 \\ \text { SSp-II } & 28 \\ \text { SSp-tr } & 29 \\ \text { SSp-ul } & 30 \\ \text { TEa } & 31 \\ \text { VISli } & 32 \\ \text { AUDp } & 33 \\ \text { AUDv } & 34 \\ \text { AUDd } & 35 \\ \text { AUDpo } & \end{array}$

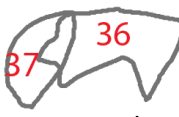

bregma - $0.94 \mathrm{~mm}$
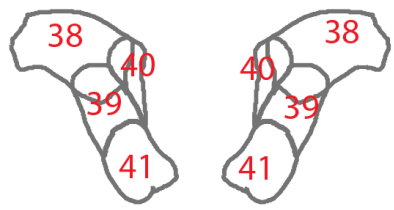

bregma $-2.46 \mathrm{~mm}$

Posterior parietal association areas

PTLp

Rostrolateral visual area

VISrl

Anterior area

VISa

Postrhinal area

VISpor

Lateral visual area

VISI

Posterolateral visual area

VISpl

Anterolateral visual area

VISal

Ectorhinal area

ECT

Entorhinal area

ENT

Presubiculum

Postsubiculum

PRE

Area prostriata

Primary visual area

Apr

Anteromedial visual area

VISp

VISam

posteromedial visual area

VISpm

RSPd

RSPV

Retrosplenial area, ventral part

RSPagl

$\begin{array}{ll}\text { LP } & 39 \\ \text { LGd } & 40 \\ \text { LD } & 41\end{array}$

Anteroventral nucleus of thalamus

AV Anterodorsal nucleus

AD

Anteromedial nucleus

Figure S3. Brain segmentation and nomenclature according to the Allen CCFv3. 
bioRxiv preprint doi: https://doi.org/10.1101/2021.01.31.429033; this version posted August 2, 2021. The copyright holder for this preprint (which was not certified by peer review) is the author/funder, who has granted bioRxiv a license to display the preprint in perpetuity. It is made available under aCC-BY 4.0 International license.

\begin{tabular}{|c|c|c|c|c|c|c|c|c|c|c|c|}
\hline $\begin{array}{l}\text { Number of input cells } \\
\text { thalamus }\end{array}$ & average & sd & sample 1 & sample 2 & sample 3 & & & & & & \\
\hline LP & 1191 & 191 & 1409 & 1052 & 1111 & & & & & & \\
\hline ATN & 1001 & 417 & 1465 & 657 & 882 & & & & & & \\
\hline LGd & 194 & 174 & 366 & 198 & 19 & \multicolumn{6}{|c|}{ Layerwise contribution } \\
\hline proximal cortex & & & & & & L1 & L2/3 & L4 & L5 & L6a & L6b \\
\hline VISp & 1245 & 282 & 954 & 1262 & 1518 & $1.83 \%$ & $39.36 \%$ & $8.10 \%$ & $42.97 \%$ & $5.97 \%$ & $1.77 \%$ \\
\hline VISpm & 842 & 264 & 687 & 692 & 1146 & $0.28 \%$ & $16.63 \%$ & $14.62 \%$ & $55.98 \%$ & $9.68 \%$ & $2.80 \%$ \\
\hline VISam & 418 & 227 & 234 & 348 & 672 & $0.00 \%$ & $15.12 \%$ & $10.75 \%$ & $60.37 \%$ & $12.43 \%$ & $1.32 \%$ \\
\hline VISI & 334 & 144 & 170 & 392 & 439 & $0.17 \%$ & $25.97 \%$ & $20.55 \%$ & $46.06 \%$ & $5.92 \%$ & $1.33 \%$ \\
\hline VISal & 118 & 65 & 46 & 174 & 133 & $0.00 \%$ & $20.08 \%$ & $23.55 \%$ & $50.62 \%$ & $5.36 \%$ & $0.40 \%$ \\
\hline RSPv & 1825 & 844 & 2705 & 1022 & 1747 & $0.29 \%$ & $11.40 \%$ & $n / a$ & $70.43 \%$ & $17.87 \%$ & $0.01 \%$ \\
\hline RSPd & 1038 & 314 & 1256 & 678 & 1180 & $0.29 \%$ & $9.97 \%$ & $n / a$ & $65.15 \%$ & $24.45 \%$ & $0.14 \%$ \\
\hline RSPagl & 785 & 353 & 967 & 378 & 1010 & $1.14 \%$ & $24.25 \%$ & $\mathrm{n} / \mathrm{a}$ & $64.46 \%$ & $9.96 \%$ & $0.19 \%$ \\
\hline \multicolumn{12}{|l|}{ distal cortex } \\
\hline ORB & 305 & 117 & 181 & 320 & 414 & $19.25 \%$ & $72.86 \%$ & $\mathrm{n} / \mathrm{a}$ & $6.22 \%$ & $1.67 \%$ & $0.00 \%$ \\
\hline ACA & 252 & 161 & 189 & 131 & 435 & $0.96 \%$ & $12.20 \%$ & $n / a$ & $73.28 \%$ & $13.56 \%$ & $0.00 \%$ \\
\hline PTLp & 221 & 87 & 120 & 266 & 276 & $0.00 \%$ & $11.16 \%$ & $10.62 \%$ & $65.23 \%$ & $12.60 \%$ & $0.39 \%$ \\
\hline AUD & 177 & 28 & 145 & 195 & 192 & $1.58 \%$ & $17.60 \%$ & $10.83 \%$ & $60.17 \%$ & $8.18 \%$ & $1.64 \%$ \\
\hline
\end{tabular}

741 Supplementary table 1. Results of rabies tracing experiments. 

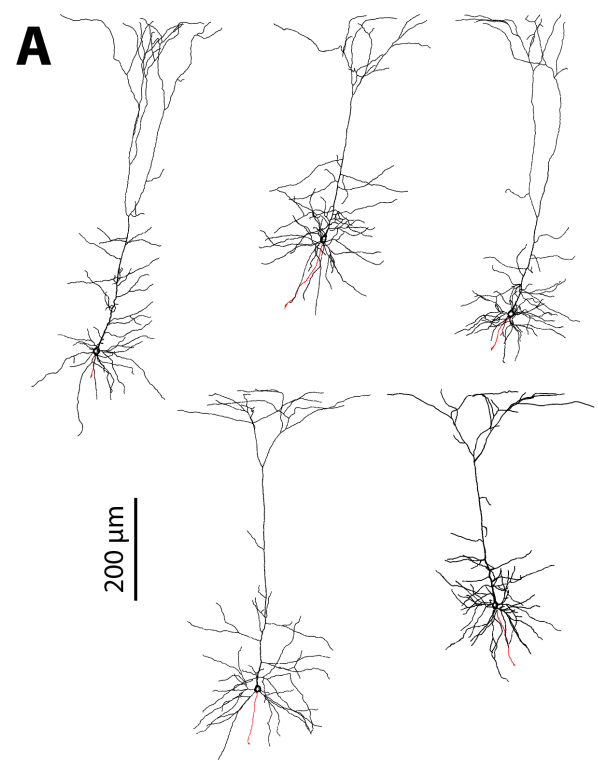

B
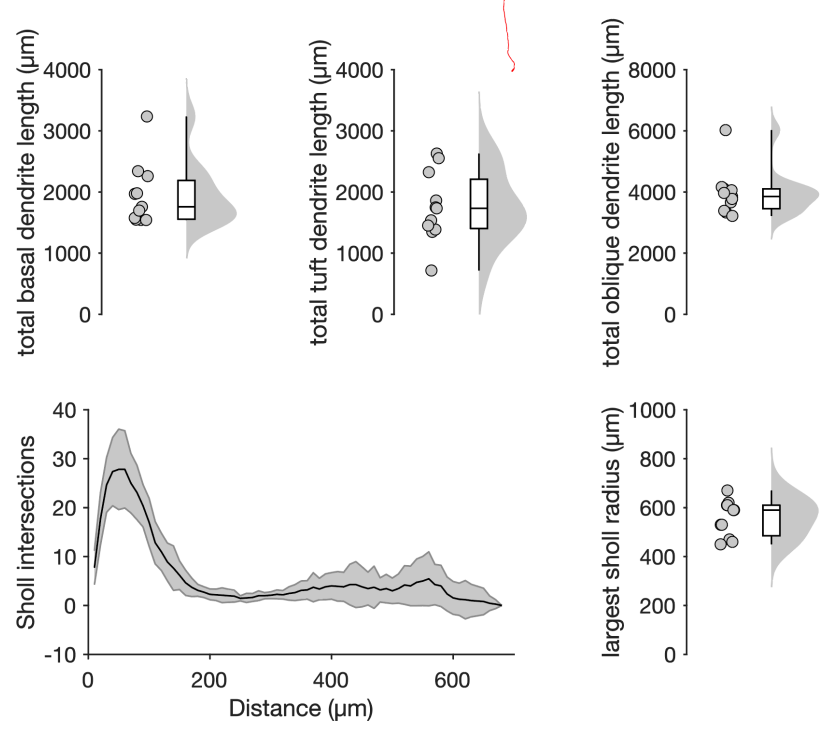

743

Figure S4. A. Reconstructed morphologies of 11 Colgalt-2 neurons. Black: dendrites; red: axons. B. Quantitative descriptive measures of dendritic morphology. 
bioRxiv preprint doi: https://doi.org/10.1101/2021.01.31.429033; this version posted August 2, 2021. The copyright holder for this preprint (which was not certified by peer review) is the author/funder, who has granted bioRxiv a license to display the preprint in perpetuity. It is made available under aCC-BY 4.0 International license.

A

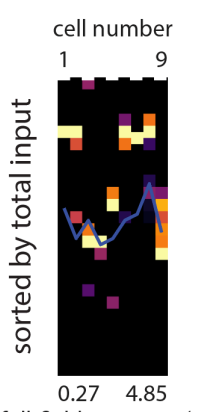

full-field response $(p c)$

C

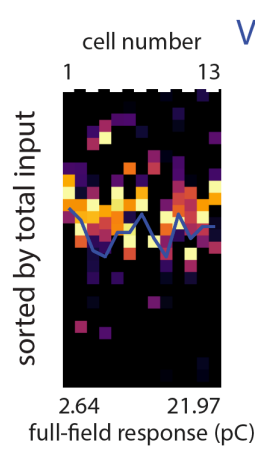

$\mathrm{E}$

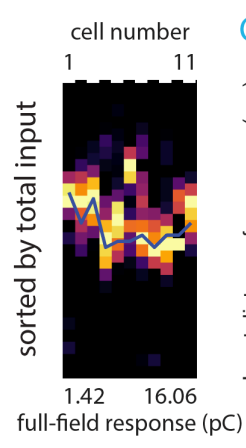

$\mathrm{VISp} \rightarrow \mathrm{V} 2 \mathrm{M}$

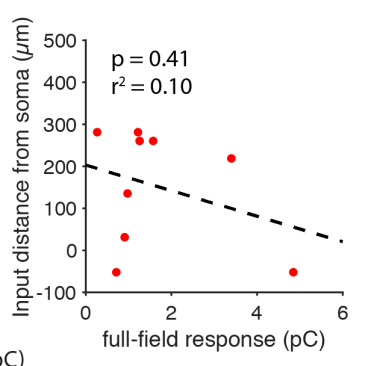

$\mathrm{V} 2 \mathrm{M} \rightarrow \mathrm{V} 2 \mathrm{M}$

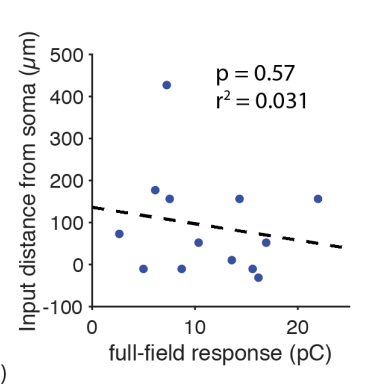

$\mathrm{ORB} \rightarrow \mathrm{V} 2 \mathrm{M}$

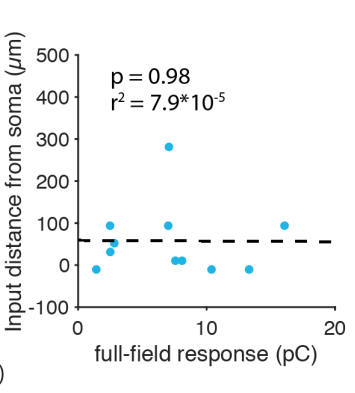

B

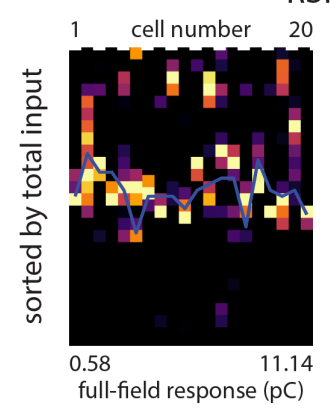

$\mathrm{RSPg} \rightarrow \mathrm{V} 2 \mathrm{M}$

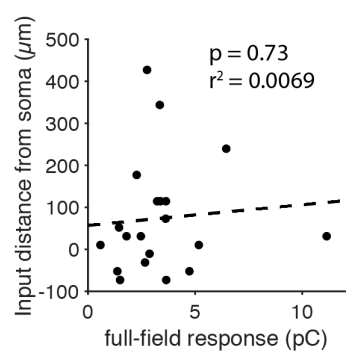

D
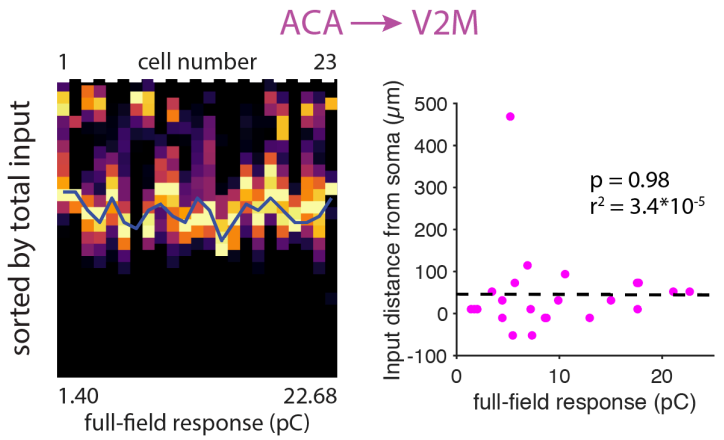

F

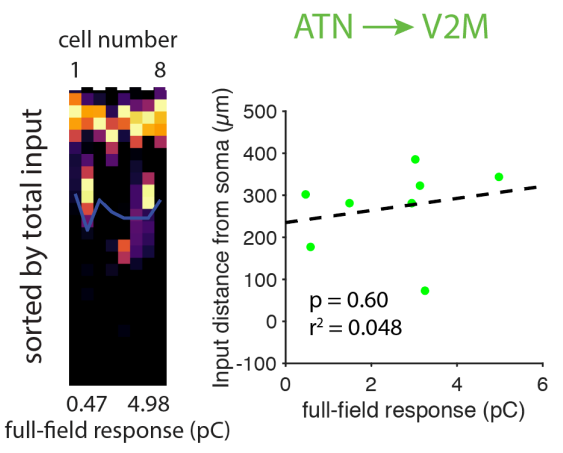

G

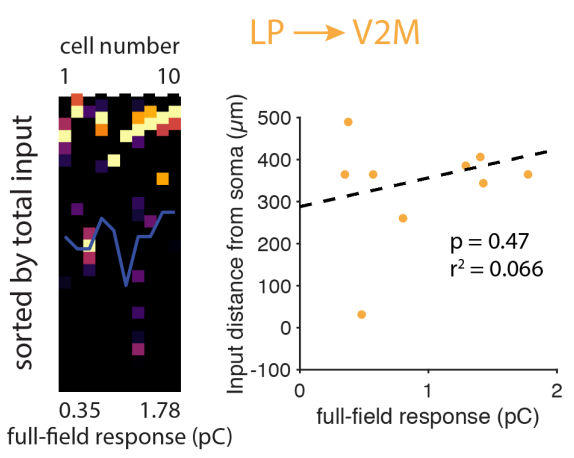

Figure S5. Input location does not correlate with input strength. A. Horizontal projections of VISp input to individual cells sorted by full-field stimulation response. Right: Location of the largest input peak versus full-field response. Dashed line is a linear fit. B-G. Same as in A, but for RSPg, V2M, ACA, ORB, ATN and LP input. 


\begin{tabular}{|c|c|c|c|c|c|c|}
\hline input area & parameter & basal & oblique & tuft* & & \\
\hline VISp & peak location $(\mu \mathrm{m})$ & -62.5 & 104.17 & 187.5 & total input $(\mathrm{pC})$ & 0.93 \\
\hline$N=6$ & input proportion (\%) & $26 \%$ & $33 \%$ & $42 \%$ & total input SEM & 0.11 \\
\hline \multirow[t]{2}{*}{$n=9$} & proportional input $(\mathrm{pC})$ & 0.24 & 0.30 & 0.39 & soma depth $(\mu \mathrm{m})$ & $507 \pm 22$ \\
\hline & horizontal bias $(\mu \mathrm{m}){ }^{* \star}$ & 62.5 & 41.67 & -20.83 & cells with peak in tuft & $5 / 9$ \\
\hline V2M & peak location $(\mu \mathrm{m})$ & -41.67 & 83.33 & 166.67 & total input $(p C)$ & 11.24 \\
\hline$N=4$ & input proportion (\%) & $24 \%$ & $62 \%$ & $14 \%$ & total input SEM & 1.56 \\
\hline \multirow[t]{2}{*}{$n=13$} & proportional input $(\mathrm{pC})$ & 2.68 & 6.96 & 1.60 & soma depth $(\mu \mathrm{m})$ & $498 \pm 15$ \\
\hline & horizontal bias $(\mu \mathrm{m})^{* \star}$ & 20.83 & 20.83 & -20.83 & cells with peak in tuft & $1 / 13$ \\
\hline RSPg & peak location $(\mu \mathrm{m})$ & -41.67 & 41.67 & 125 & total input $(\mathrm{pC})$ & 3.40 \\
\hline$N=9$ & input proportion (\%) & $30 \%$ & $40 \%$ & $30 \%$ & total input SEM & 0.51 \\
\hline \multirow[t]{2}{*}{$n=20$} & proportional input $(\mathrm{pC})$ & 1.04 & 1.36 & 1.01 & soma depth $(\mu \mathrm{m})$ & $503 \pm 15$ \\
\hline & horizontal bias $(\mu \mathrm{m}){ }^{\star \star}$ & 20.83 & 20.83 & 0 & cells with peak in tuft & $2 / 20$ \\
\hline ACA & peak location $(\mu \mathrm{m})$ & -41.67 & 41.67 & 83.33 & total input $(\mathrm{pC})$ & 9.46 \\
\hline$N=5$ & input proportion (\%) & $30 \%$ & $45 \%$ & $25 \%$ & total input SEM & 1.32 \\
\hline \multirow[t]{2}{*}{$n=23$} & proportional input $(\mathrm{pC})$ & 2.83 & 4.22 & 2.41 & soma depth $(\mu \mathrm{m})$ & $464 \pm 9$ \\
\hline & horizontal bias $(\mu \mathrm{m})^{* \star}$ & 0 & 0 & -20.83 & cells with peak in tuft & $1 / 23$ \\
\hline ORB & peak location $(\mu \mathrm{m})$ & -41.67 & 20.83 & $N / A$ & total input $(\mathrm{pC})$ & 7.16 \\
\hline$N=3$ & input proportion (\%) & $35 \%$ & $57 \%$ & $9 \%$ & total input SEM & 1.43 \\
\hline \multirow[t]{2}{*}{$n=11$} & proportional input (pC) & 2.47 & 4.05 & 0.63 & soma depth $(\mu \mathrm{m})$ & $521 \pm 10$ \\
\hline & horizontal bias $(\mu \mathrm{m}){ }^{* \star}$ & 0 & 20.83 & 0 & cells with peak in tuft & $0 / 11$ \\
\hline ATN & peak location $(\mu \mathrm{m})$ & -104.17 & 104.17 & 104.17 & total input $(\mathrm{pC})$ & 2.48 \\
\hline$N=3$ & input proportion (\%) & $8 \%$ & $17 \%$ & $75 \%$ & total input SEM & 0.54 \\
\hline \multirow[t]{2}{*}{$n=8$} & proportional input $(\mathrm{pC})$ & 0.21 & 0.42 & 1.86 & soma depth $(\mu \mathrm{m})$ & $435 \pm 15$ \\
\hline & horizontal bias $(\mu \mathrm{m}){ }^{* *}$ & 20.83 & 41.67 & 20.83 & cells with peak in tuft & $6 / 8$ \\
\hline LP & peak location $(\mu \mathrm{m})$ & -41.67 & 20.83 & 62.5 & total input $(\mathrm{pC})$ & 0.97 \\
\hline$N=4$ & input proportion (\%) & $10 \%$ & $15 \%$ & $75 \%$ & total input SEM & 0.16 \\
\hline \multirow[t]{3}{*}{$n=10$} & proportional input $(\mathrm{pC})$ & 0.10 & 0.14 & 0.72 & soma depth $(\mu \mathrm{m})$ & $500 \pm 23$ \\
\hline & horizontal bias $(\mu \mathrm{m}){ }^{* \star}$ & -41.67 & -20.83 & -83.33 & cells with peak in tuft & $9 / 10$ \\
\hline & total proportional input & $27 \%$ & $49 \%$ & $24 \%$ & sum total input (pC) & 35.65 \\
\hline
\end{tabular}

*tuft measurements from pia, basal and oblique from soma

** negative means lateral, positive medial

Supplementary table 2. Results of all SCRACM experiments. 
A

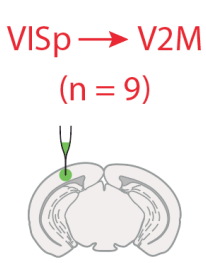

C

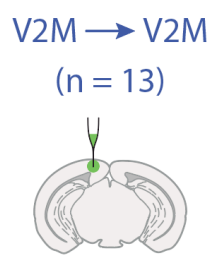

Figure S6. Detailed data analysis for VISp and V2M. A, C. Vertical projections of individual input maps sorted by the location of the peak input. B, D. Horizontal projections of individual input maps and their average for all inputs, and for domain-separated inputs. 

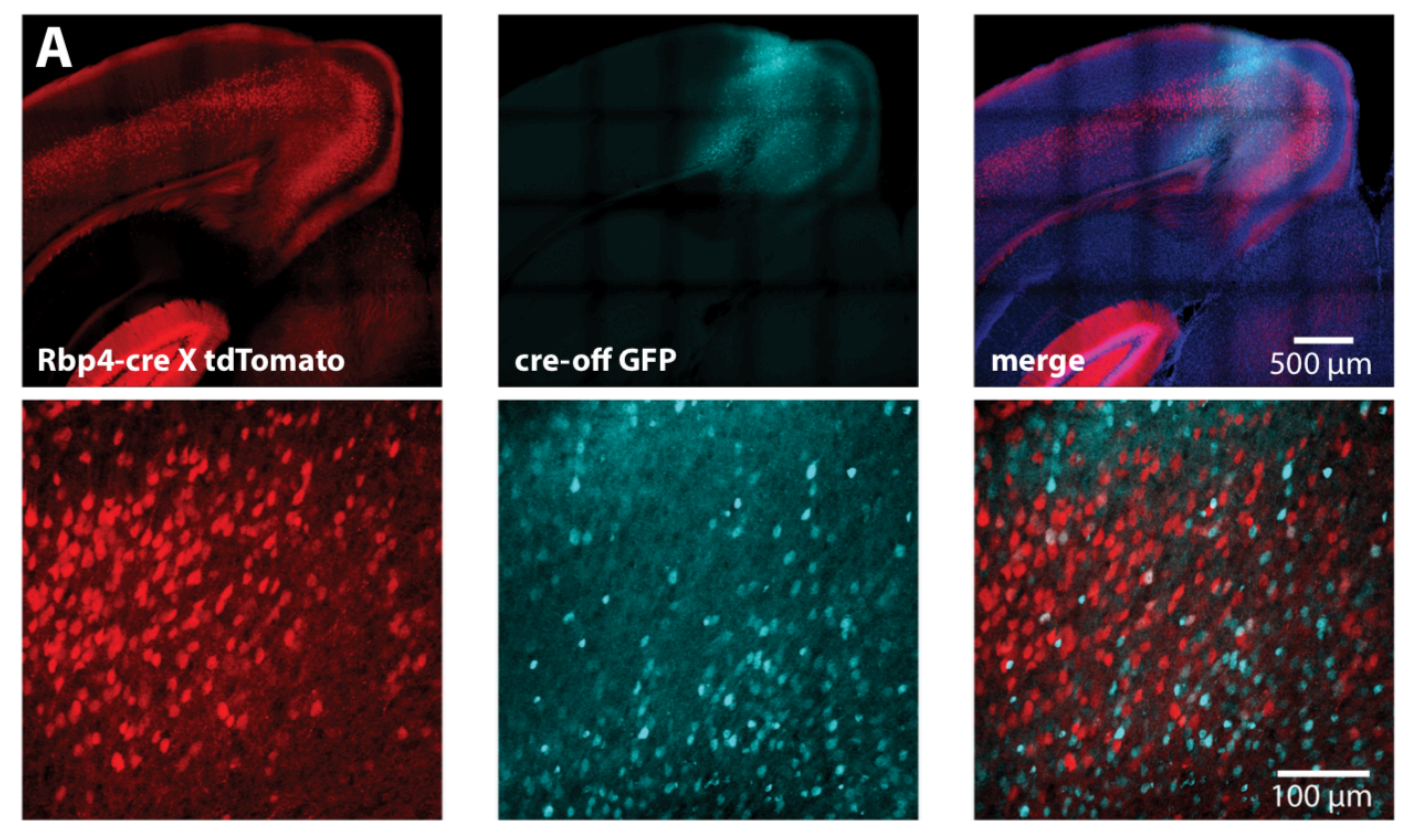

B

GFP: 127
Rbp4: 184

760 Figure S7. Efficacy of Cre-off virus. A. Example confocal images showing cre expression in L5 pyramidal neurons

761 (red), GFP expression following cre-off virus injection (cyan) and overlap (blue = DAPI) on two magnifications. B.

762 Quantification of overlap between cre and GFP expression. 
bioRxiv preprint doi: https://doi.org/10.1101/2021.01.31.429033; this version posted August 2, 2021. The copyright holder for this preprint (which was not certified by peer review) is the author/funder, who has granted bioRxiv a license to display the preprint in perpetuity. It is made available under aCC-BY 4.0 International license.

A
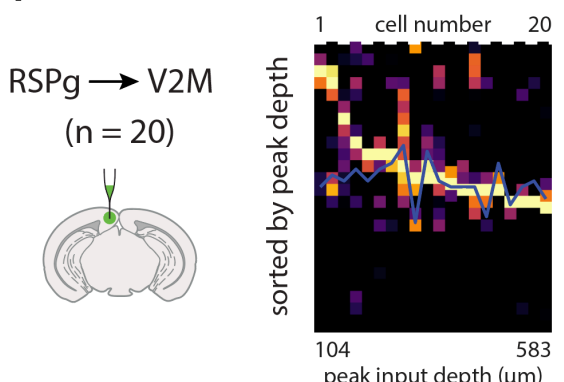

C

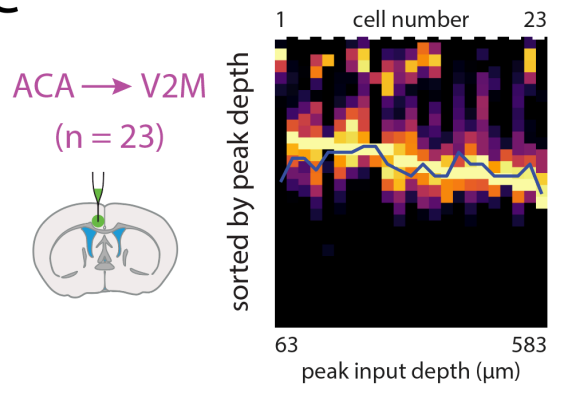

$E$

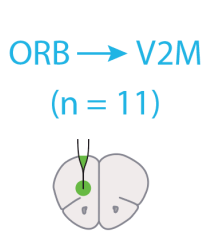

763

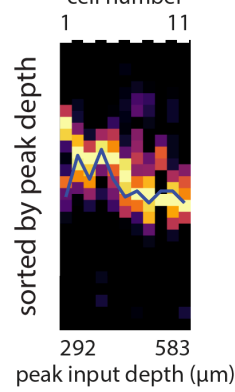
all inputs, and for domain-separated inputs.
B
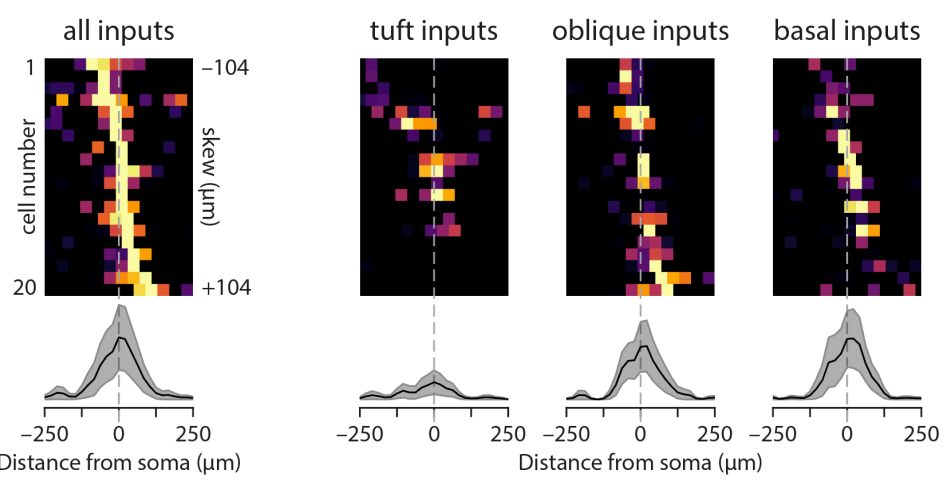

D

all inputs

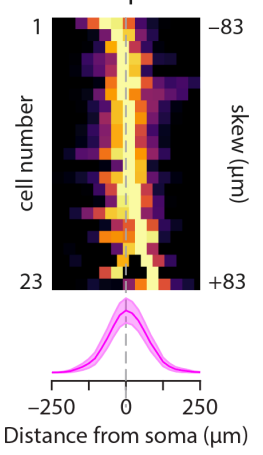

tuft inputs

oblique inputs

basal inputs
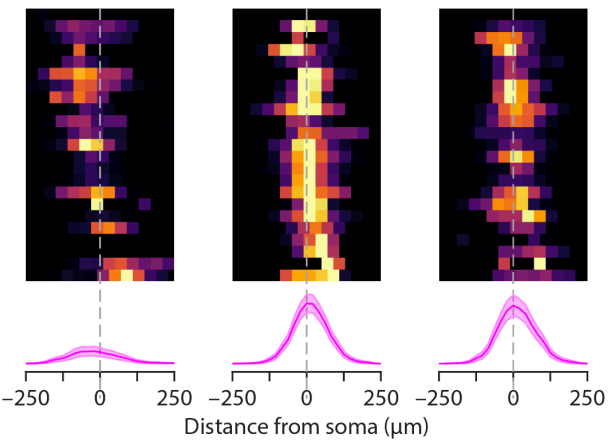

$\mathrm{F}$

all inputs

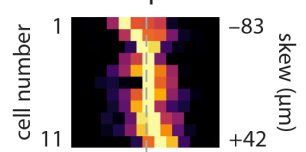

tuft inputs

oblique inputs

basal inputs
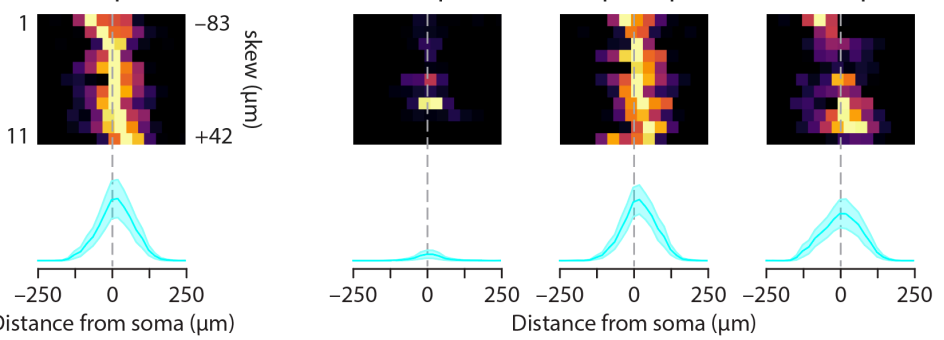

Figure S8. Detailed data analysis for RSPg, ACA and ORB. A, C, E. Vertical projections of individual input maps sorted by the location of the peak input. B, D, F. Horizontal projections of individual input maps and their average for 
A
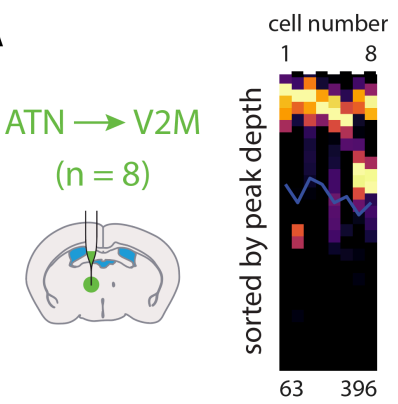

peak input depth $(\mu \mathrm{m})$

C

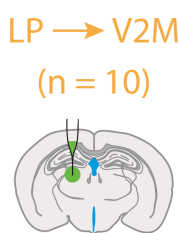

768

769

770

771 domain-separated inputs
B

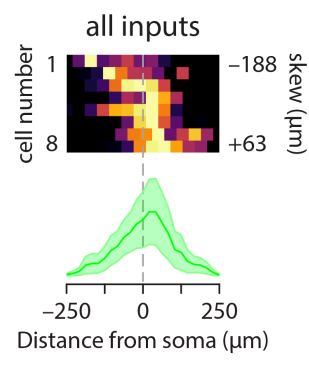

tuft inputs

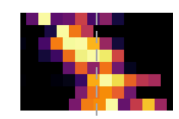

oblique inputs

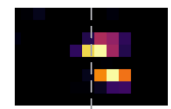

basal inputs

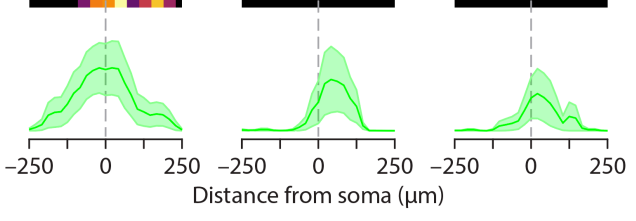

Distance from soma $(\mu \mathrm{m})$

tuft inputs

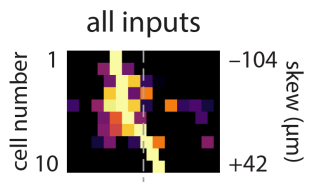

oblique inputs

basal inputs

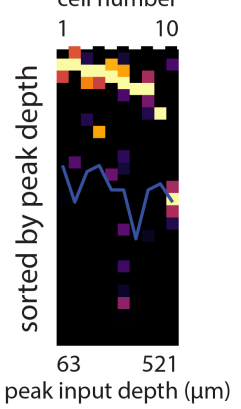

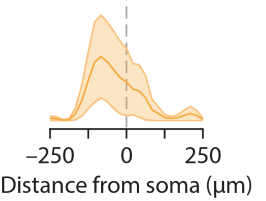
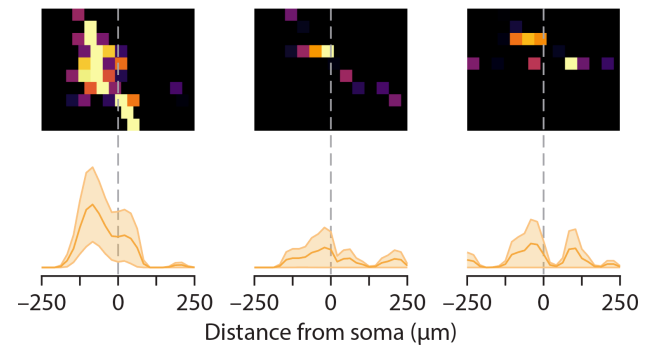

Figure S9. Detailed data analysis for ATN and LP. A, C. Vertical projections of individual input maps sorted by the location of the peak input. B, D. Horizontal projections of individual input maps and their average for all inputs, and for 
bioRxiv preprint doi: https://doi.org/10.1101/2021.0131.429033. this version posted August 2, 2021. The copyright holder for this preprint (which was not certified by peer review) is the author/funder, who has granted bioRxiv a license to display the preprint in perpetuity. It is made available under aCC-BY 4.0 International license.

$\begin{array}{lcccc}\text { Area } & \text { Distance from bregma }(\mathrm{mm}) & \text { Mediolateral distance }(\mathrm{mm}) & \text { Depth from pia }(\mathrm{mm}) & \text { Number \& volume of injections } \\ \text { VISp } & {[-3.5:-2.8]} & {[1.8: 2.7]} & {[0.5: 0.6]} & 3 \times 100 \mathrm{~nL} \\ \text { RSPg } & {[-3.2:-2.7]} & 0.5 & {[0.5: 0.7]} & 2 \times 100 \mathrm{~nL} \\ \text { V2m } & {[-3.2:-2.7]} & 1 & {[0.2: 0.5]} & 2 \times 100 \mathrm{~nL} \\ \text { ACA } & {[0: 1]} & 0.5 & {[1.2: 1.5]} & 3 \times 100 \mathrm{~nL} \\ \text { ORB } & {[2: 2.8]} & 1 & {[1.5: 2.3]} & 3 \times 100 \mathrm{~nL} \\ \text { ATN } & {[-0.5:-1.2]} & {[0.5: 0.7]} & {[3.2: 3.3]} & 2 \times 100 \mathrm{~nL} \\ \text { LP } & {[-2.5:-1.7]} & {[1: 1.5]} & {[2.4: 2.6]} & 3 \times 100 \mathrm{~nL}\end{array}$

Supplementary table 3. Stereotaxic coordinates and volumes of viral injections. 